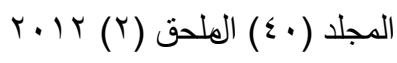

\section{تأثير بعض نظم التظذية والمعاملة الهرمونية في الأداء التناسلي ونمو الحملان للنعاج العواسية

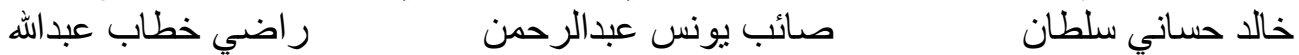 قَسم الثروة الحيو انية/كلية الزراعة والغابات/جامعة الموصل العراق}

\section{الخلاصة}

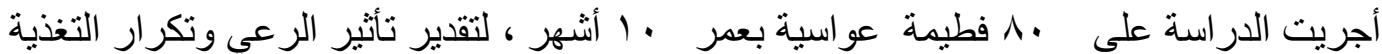

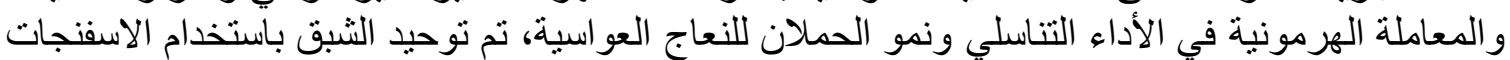

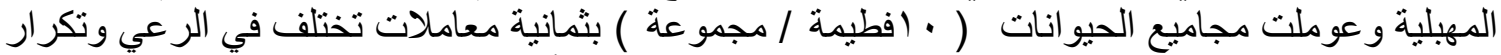

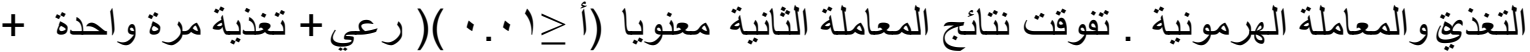

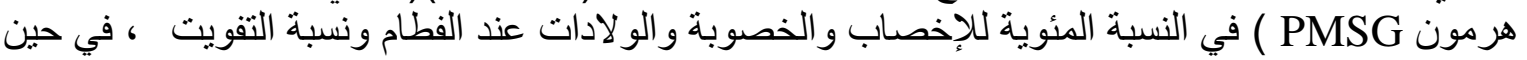

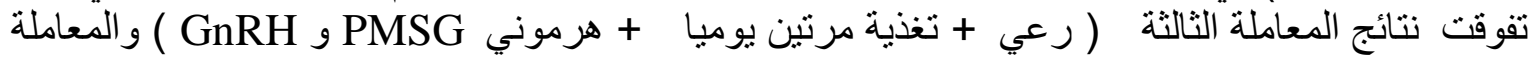

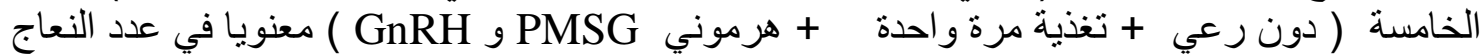

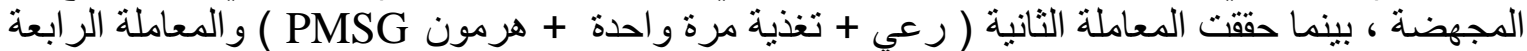

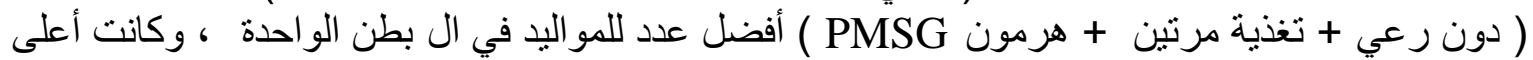

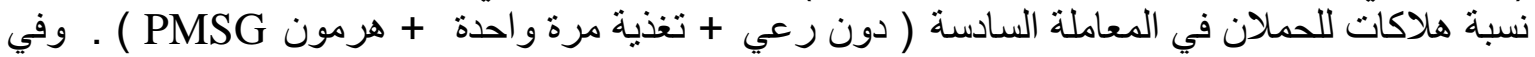

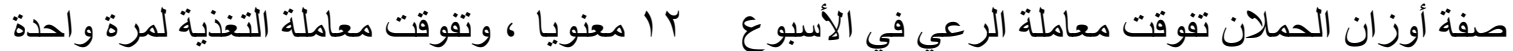

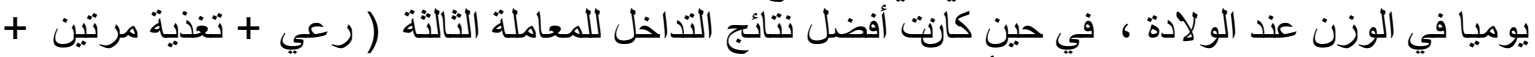

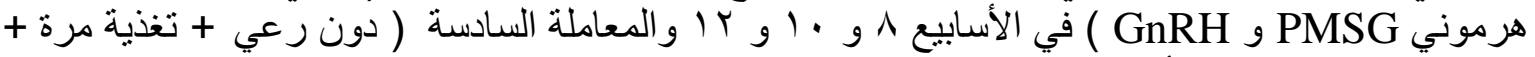

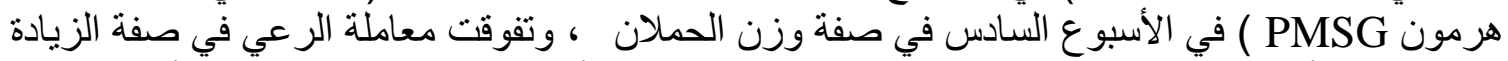

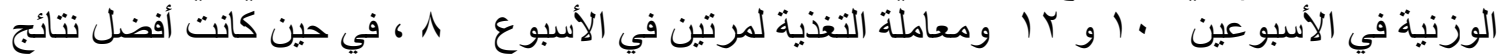

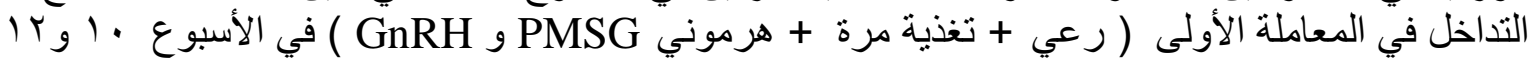

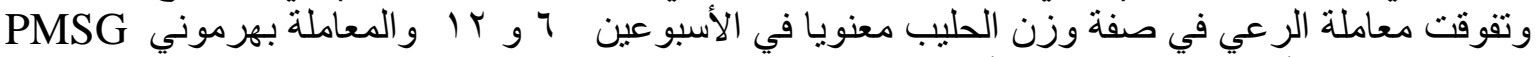

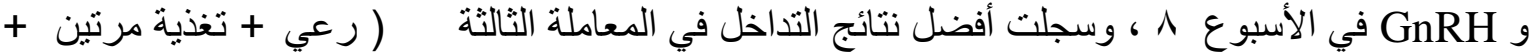

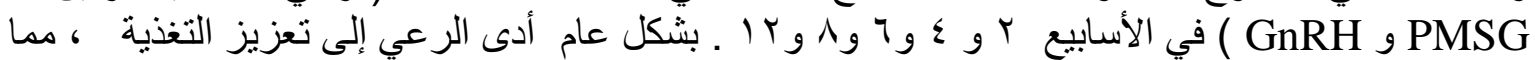
أدى إلى تحسين الأداء التناسلي للنعاج و إنتاج حليب كافي لنمو المو اليد

\section{المقدمة}

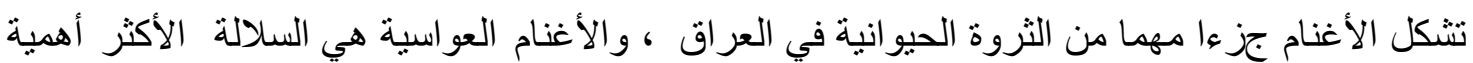

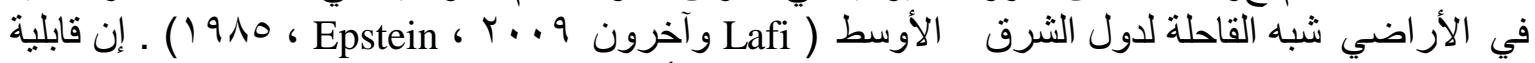

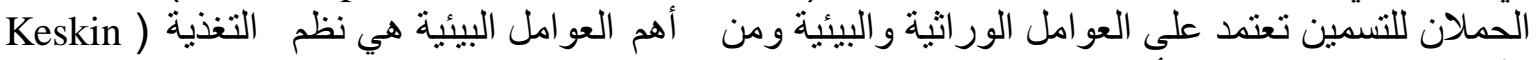

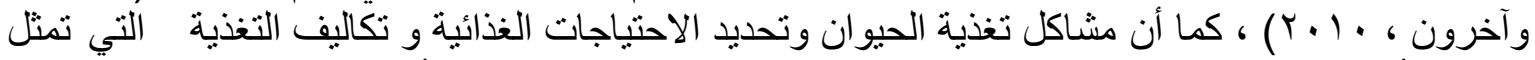

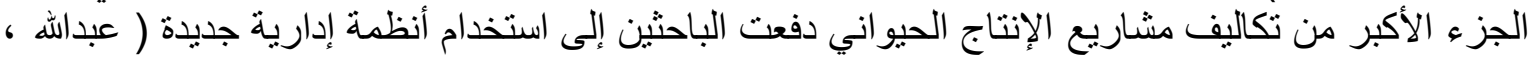

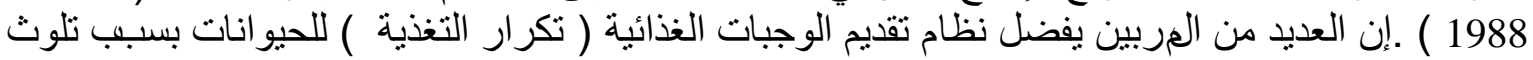

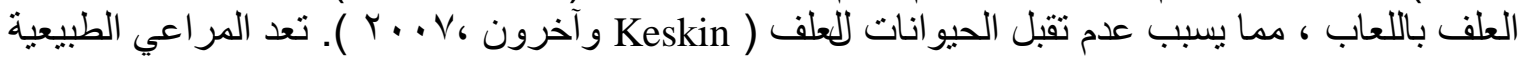

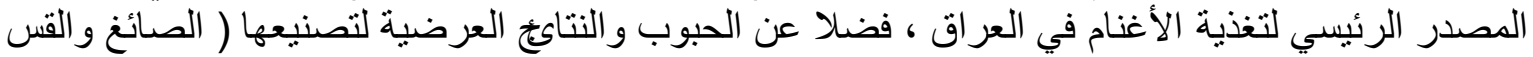

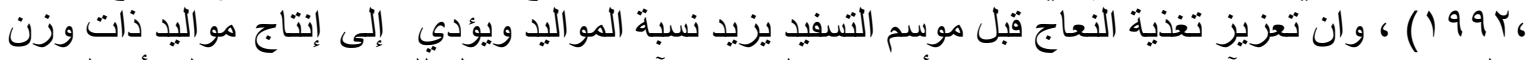

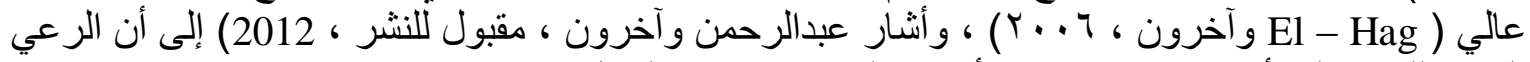

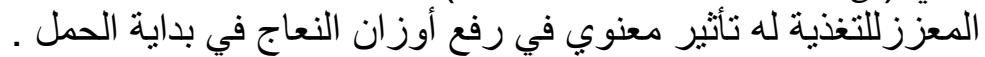

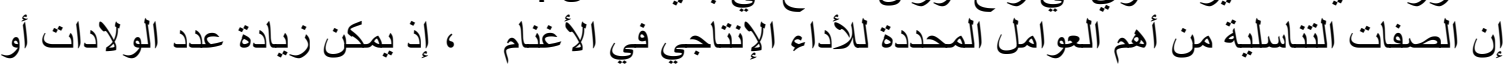

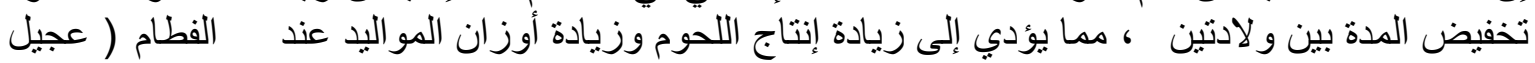

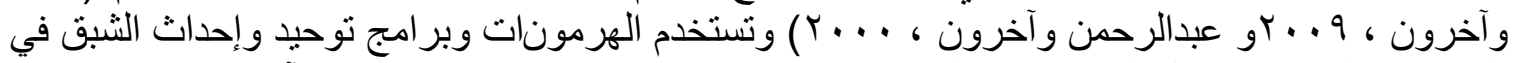
Chao

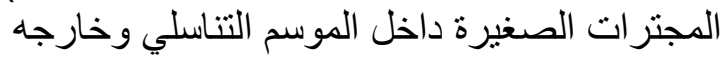




\section{～ISSN: 2224-9796 (Online)

الع ل Ungerfeld

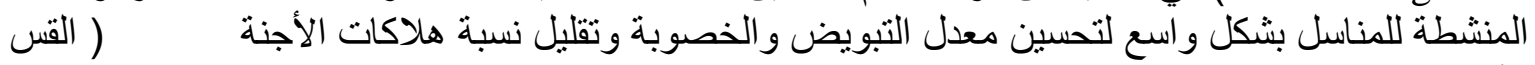

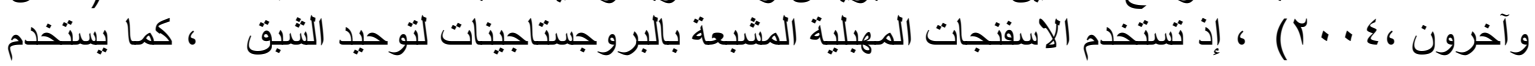

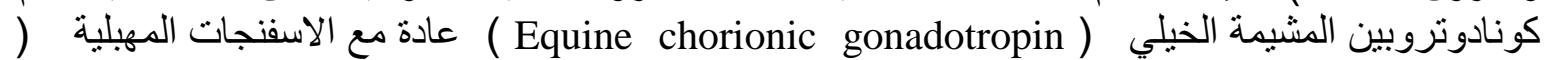

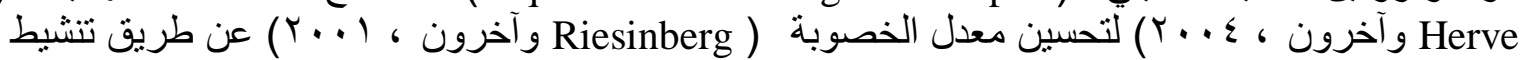

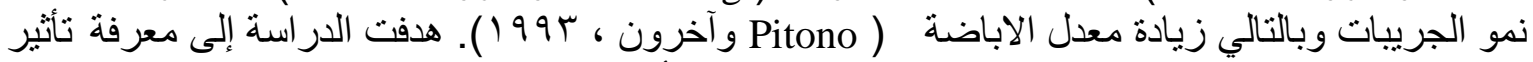

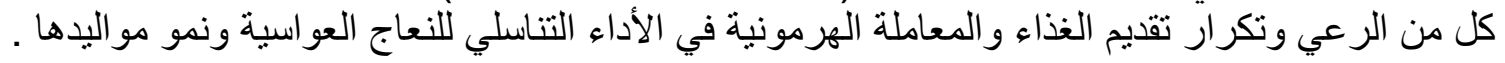

\section{مواد البحث وطرائقه}

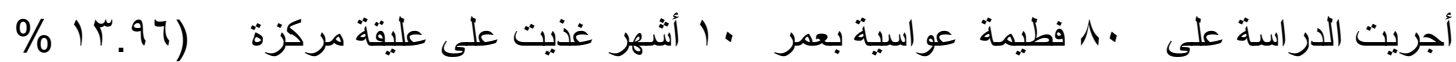

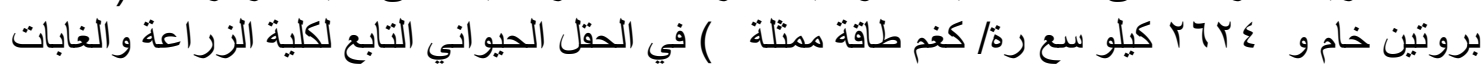

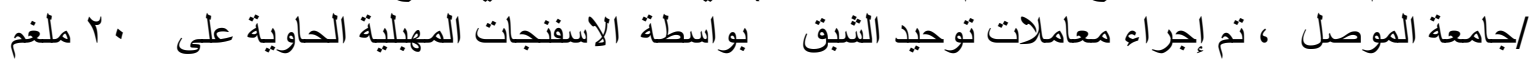

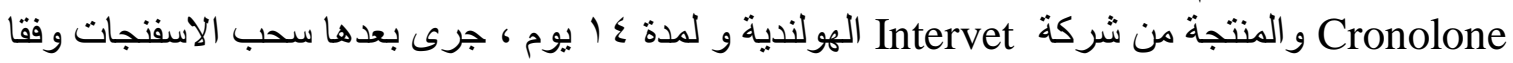

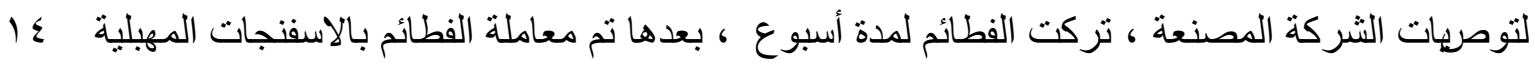

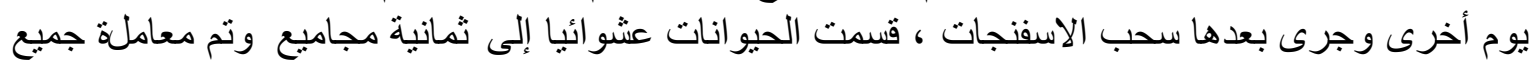

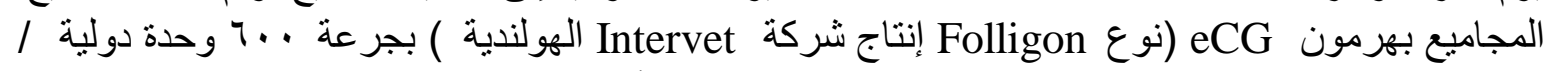

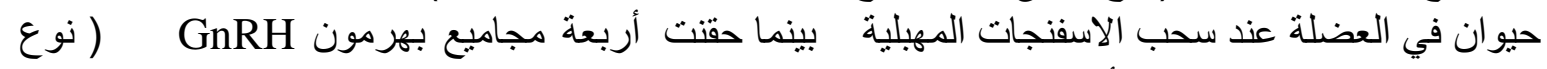

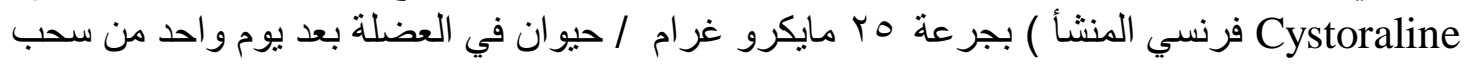

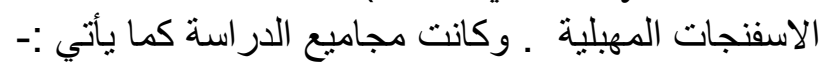

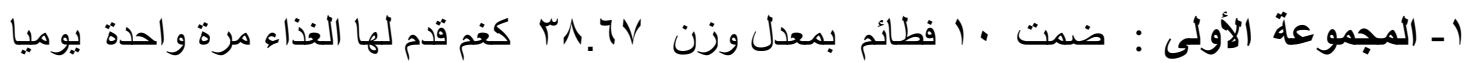

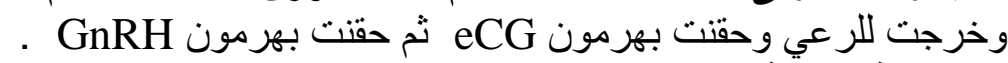

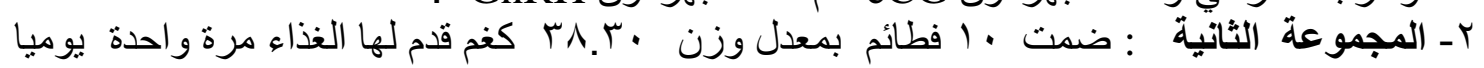

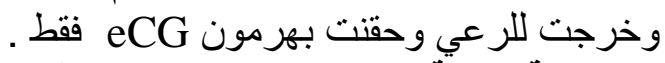

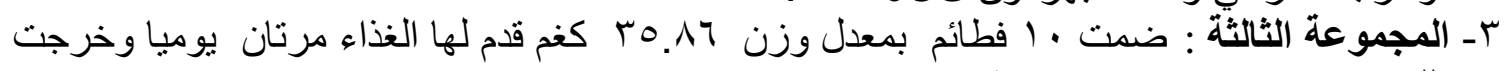

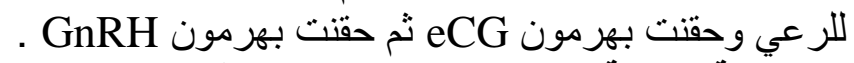

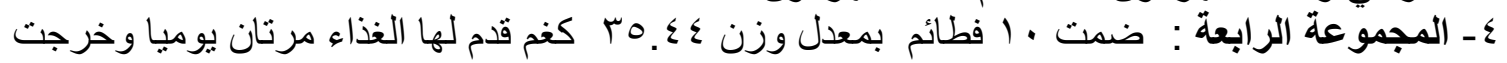

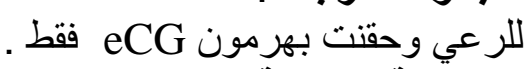

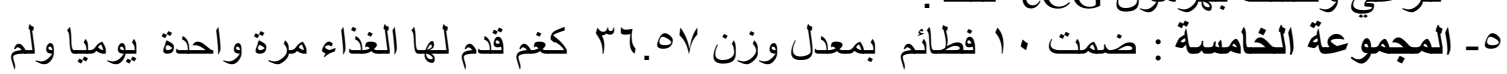

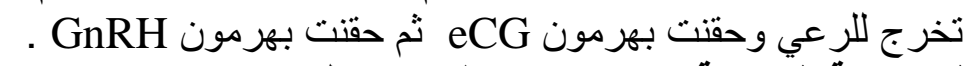

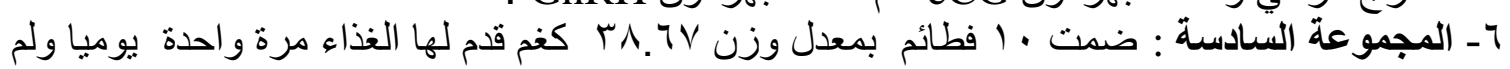

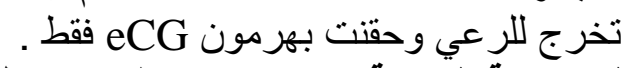

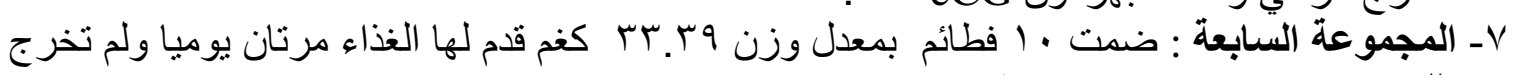

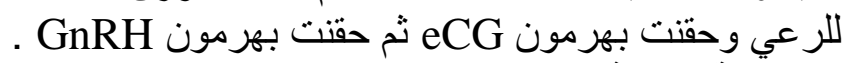

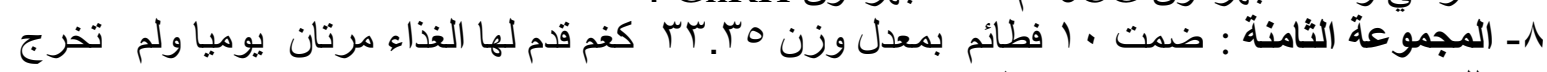

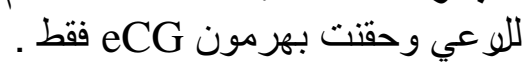
قدم الغذاء بشكل مفتوح ( ad libitum ) وتم إخر اج الحيوانات إلى الرعي ( معاملات الرعاء الرعي فقط ) بمعدل

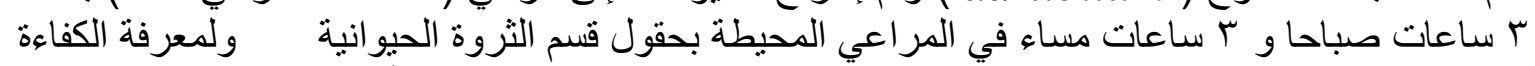

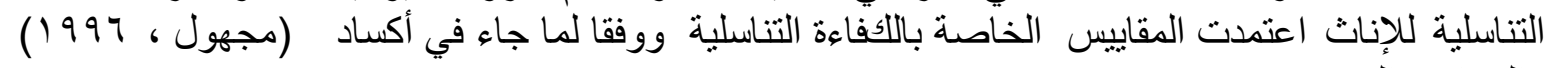

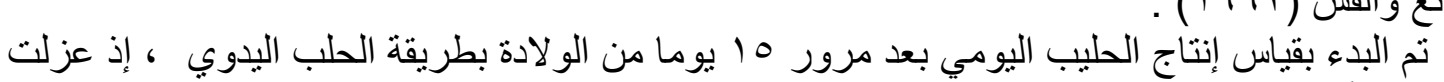

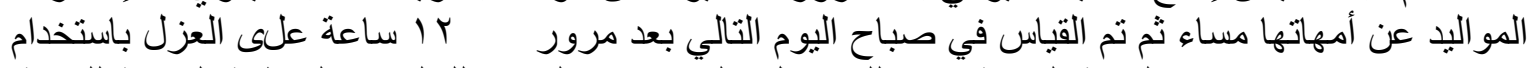

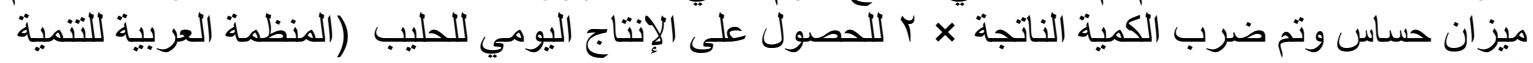
الزراعية ، 1990 (19 ) ) . 
حلت النتائج باستخدام التصميم العشو ائي الكامل(CRD) وباستخدام تجربة عاملية ذات ثلاثثة عوامل في هذه

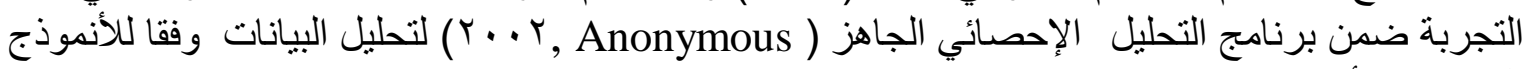
Yijkl $=\mu+\mathrm{Gi}+\mathrm{Fj}+\mathrm{Hk}+\mathrm{GFij}+\mathrm{GHik}+\mathrm{FHjk}+\mathrm{GFHijk}+$ eijkl

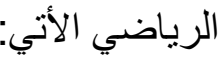

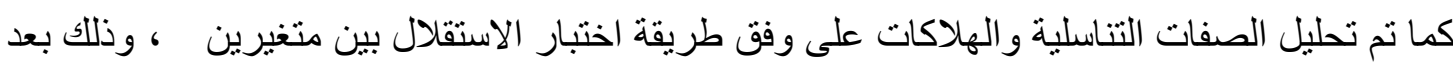

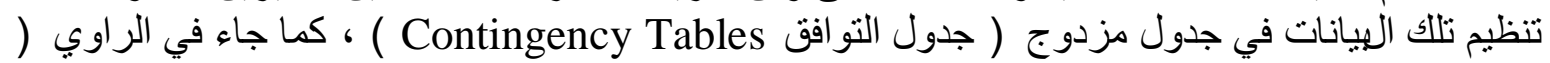

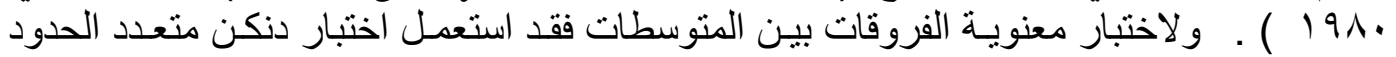

. ( $191 \leq$ ، Torrie و Steel) (Duncan's multiple range Test)

\section{النتائج والمناقشة}

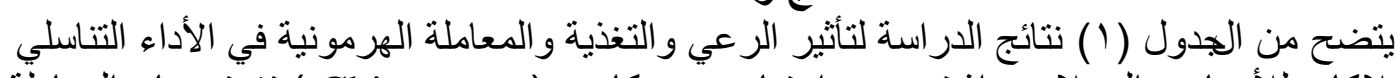

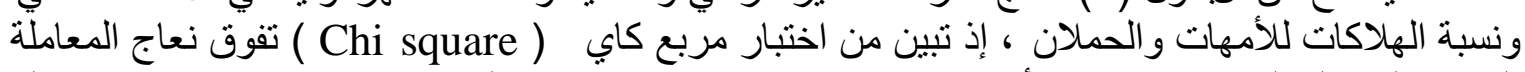

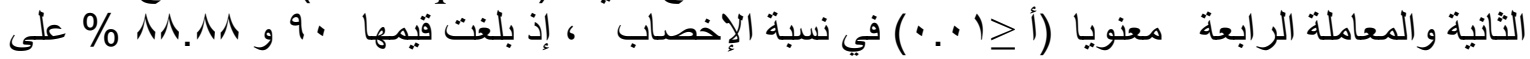

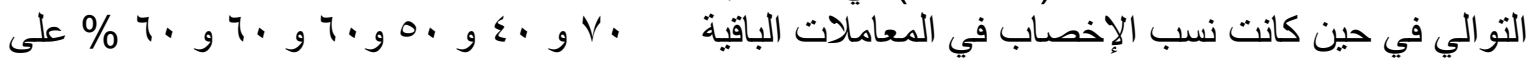

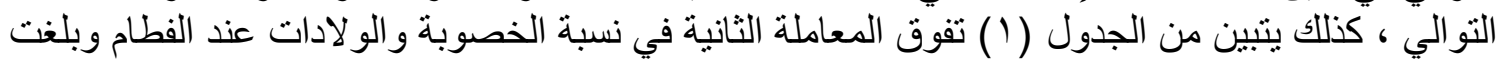

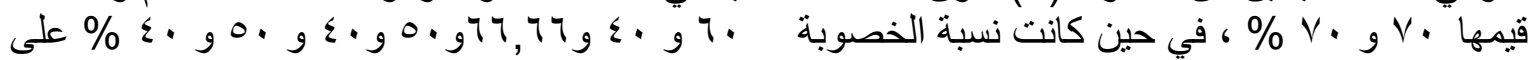

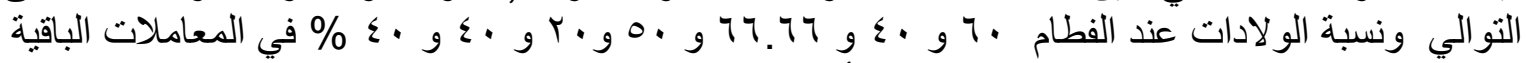

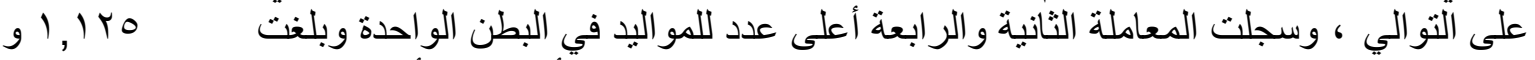

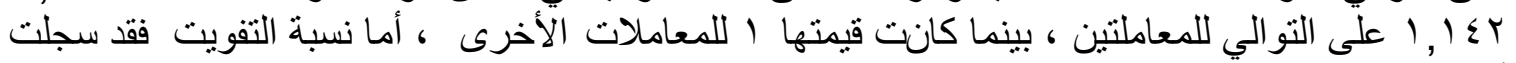

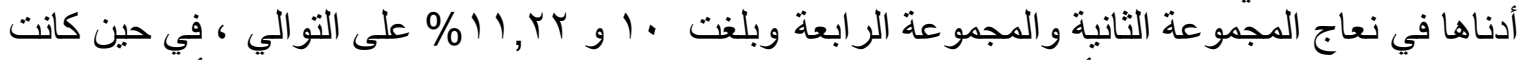

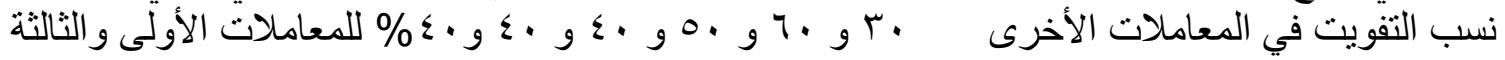

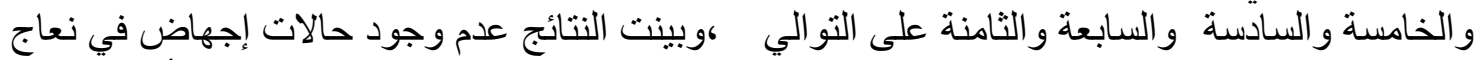

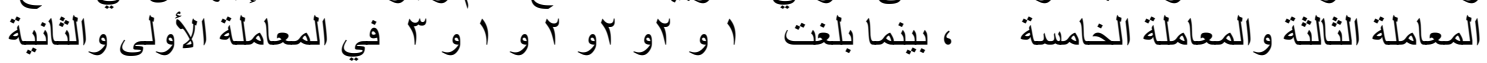

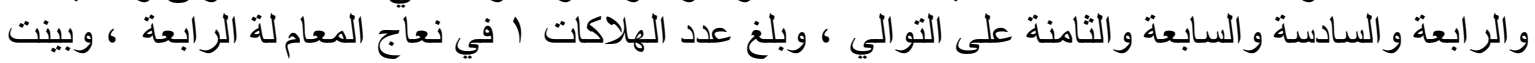

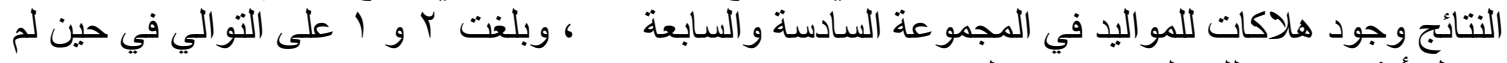

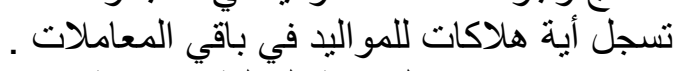

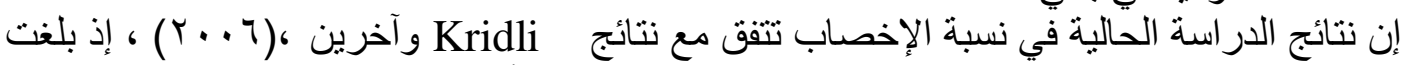

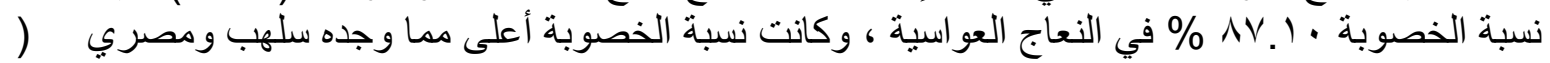

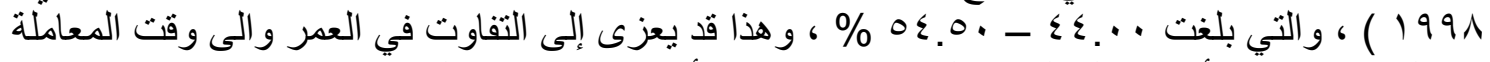

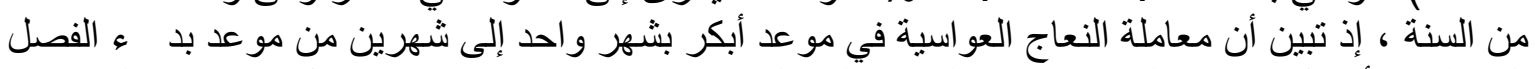

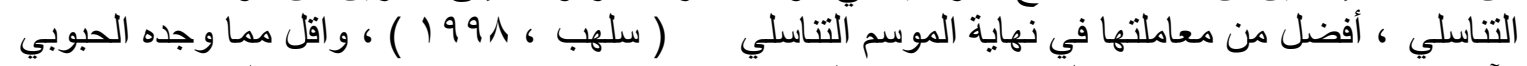

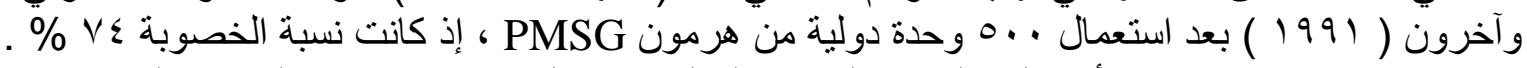

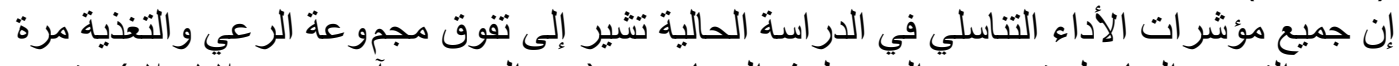

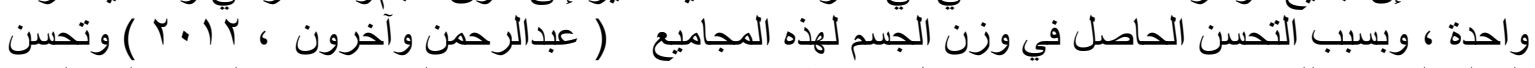

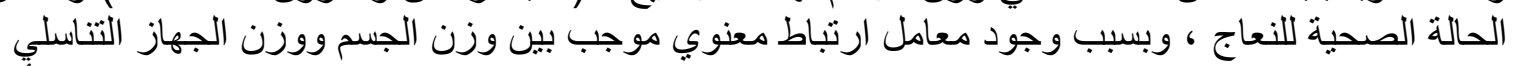

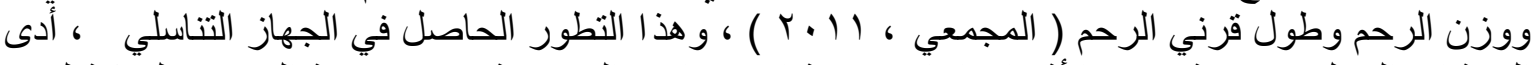

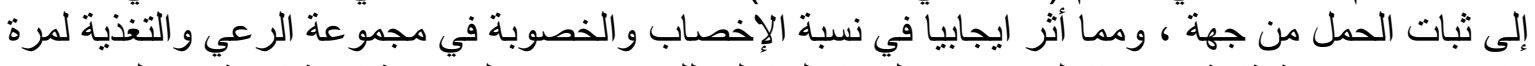

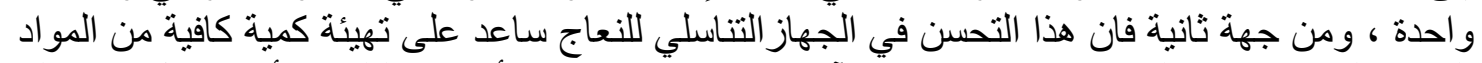

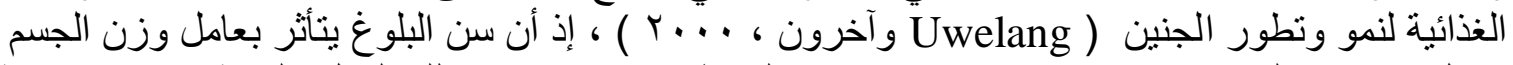

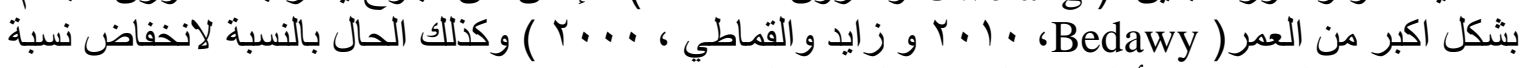

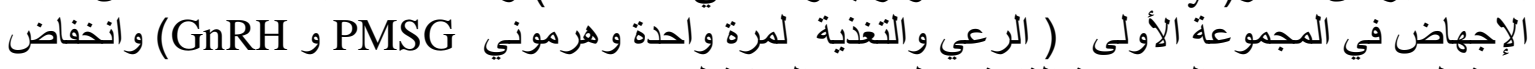

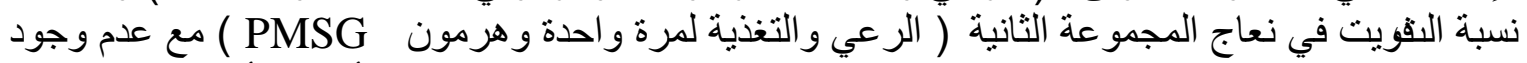

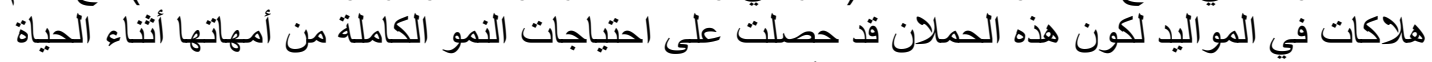

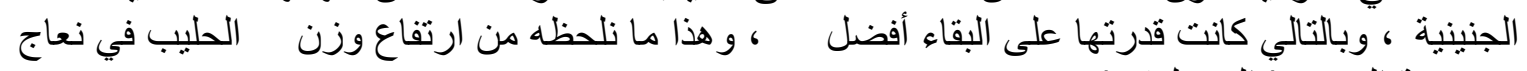

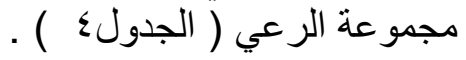




\section{ISSN: 2224-9796 (Online) \\ ISSN: $1815-316 \mathrm{X}$ (print)}

مجلة زر اعة الر افدين

الجدول( ( ) : المتوسط 土 الخطأ القياسي لتأثير الرعي و تكرار التغذية والمعاملة الهرمونية والتداخل في

\begin{tabular}{|c|c|c|c|c|c|c|c|c|}
\hline \multicolumn{2}{|c|}{ بدون رعي وتغذية } & \multicolumn{2}{|c|}{ بدون رعي وتغذية } & \multicolumn{2}{|c|}{ رعي وتغذية } & \multicolumn{2}{|c|}{ رعي وتغذية مرة واحدة } & \multirow[t]{2}{*}{ / الصفات } \\
\hline PMSG & $\begin{array}{l}\text { PMSG } \\
+\mathrm{GnRH}\end{array}$ & $\begin{array}{c}\text { PMS } \\
\text { G }\end{array}$ & $\begin{array}{l}\text { PMSG } \\
+\mathrm{GnRH}\end{array}$ & PMSG & $\begin{array}{l}\text { PMSG } \\
+\mathrm{GnRH}\end{array}$ & PMSG & $\begin{array}{l}\text { PMSG } \\
+\mathrm{GnRH}\end{array}$ & \\
\hline$\Lambda$ & V & 7 & 0 & $\varepsilon$ & $\mu$ & $r$ & 1 & رقم المعاملة \\
\hline 1. & 1. & 1. & 1. & 9 & 1. & 1 . & 1. & المعرضة للكباج \\
\hline 7 & 7 & 7 & 0 & $\wedge$ & $\varepsilon$ & 9 & V & عدد النعاج \\
\hline r & 1 & r & صفر & r & صفر & r & 1 & عدد النعاج \\
\hline$\varepsilon$ & 0 & $\varepsilon$ & 0 & 7 & $\varepsilon$ & V & 7 & عدد الحملان \\
\hline • & 1 & r & . & • & - & • & . & الهالكة للنعاج \\
\hline 7. & 7. & 7. & 0. & $\wedge \wedge . \wedge \wedge$ & $\varepsilon$. & 9. & $\vee$. & الإخصاب \\
\hline$\varepsilon$. & $\varepsilon$. & $\varepsilon$. & 0 . & $11 . K Y$ & 7. & 1. & r. & التفويت \\
\hline$\varepsilon$. & 0. & $\varepsilon$. & 0. & 77.77 & $\varepsilon$. & $\vee$. & 7. & الخصوبة \\
\hline 1 & 1 & 1 & 1 & $1.1 \leqslant r$ & 1 & 1.1 To & 1 & عدد المو اليد في البوة \\
\hline$\varepsilon$. & $\varepsilon$. & $r$. & 0. & 77.77 & $\varepsilon$. & $\vee$. & 7. & الو لادات عند الفطام \% \\
\hline - & - & - & • & 1 & • & - & • & $\begin{array}{c}\text { للأمهات الهلاكات } \\
\text { \% }\end{array}$ \\
\hline
\end{tabular}

يتضح من الجدول (Y) تفوق ولادات مجموعة الرعي في الأسبوع الثاني عشر من الو لادة في صفة الأبة

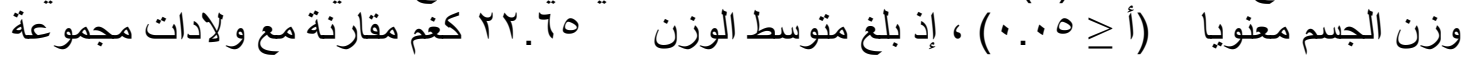

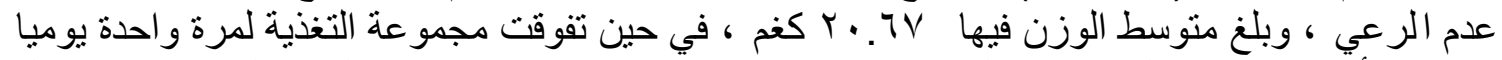

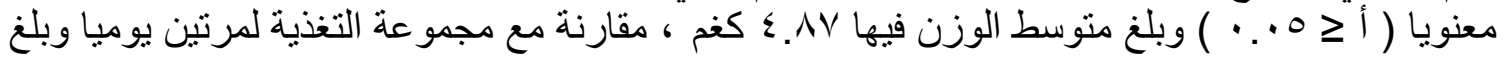

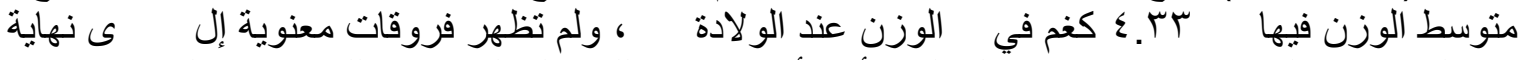

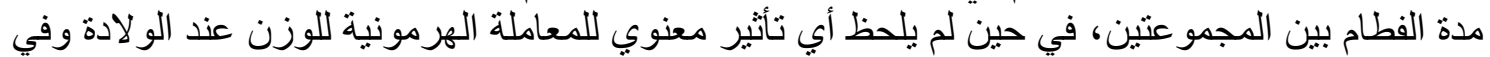

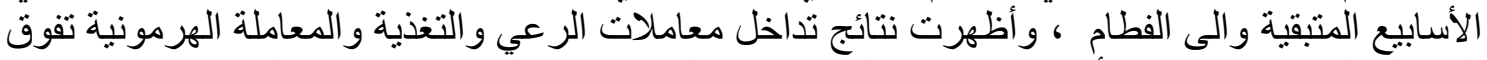

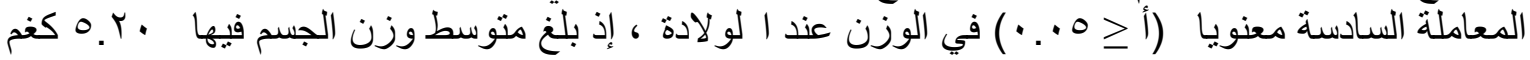

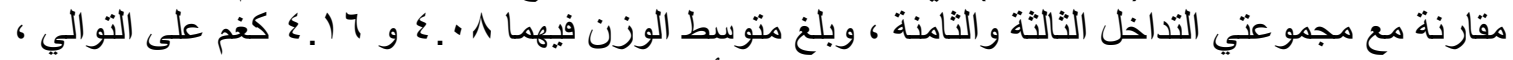

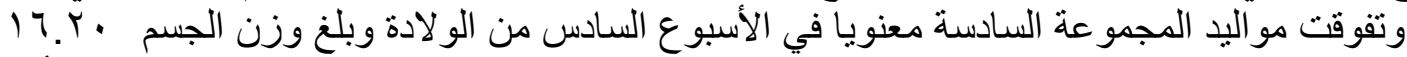

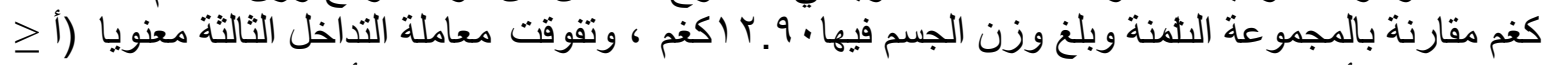

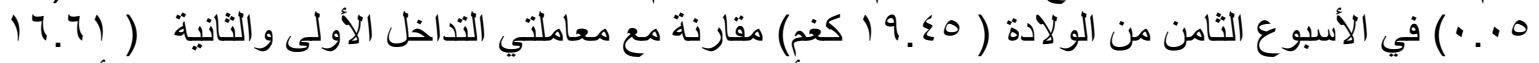

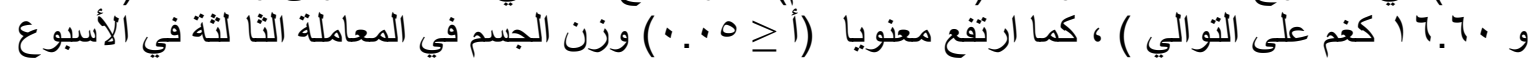

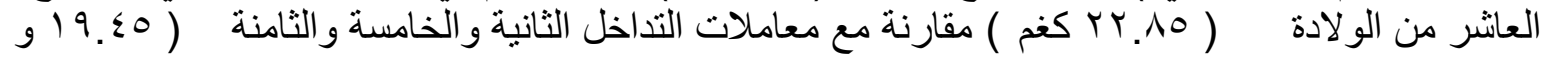




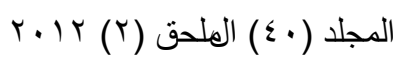
ISSN: 2224-9796 (Online)
ISSN: $1815-316 \mathrm{X}$ (print)
مجلة زر اعة الر افدين

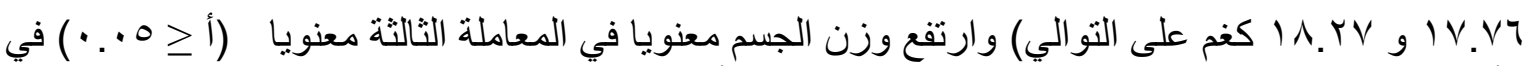

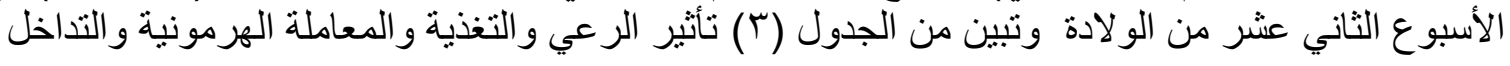

الجدول(r) : تأثثر الرعي والتغذية والمعاملة الهرمونية والتداخل في وزن الجسم ( كغ ) لحملان النعاج

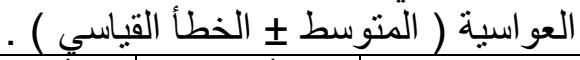

\begin{tabular}{|c|c|c|c|c|c|c|c|}
\hline الثاني عشر الأسبوع & الأسبوع & الأسبوع & السادسوع & الأسبوع & الأسبوع & 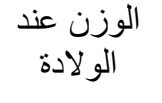 & \\
\hline \multicolumn{8}{|r|}{ * تأثثير الرعي } \\
\hline IYY.70 & سr. & IIV.\&. & IIE.YY & $11 \cdot .0 \leqslant$ & IV.ru & $|\leq .7|$ & \multirow{2}{*}{ ل اعي } \\
\hline $.0 r \pm$ & $.0 \leqslant \pm$ & $\because \leqslant 0 \pm$ & $\cdot . \leqslant \pm$ & $\cdot r \leq \pm$ & $\cdot r \mu \pm$ & $. .1 Y \pm$ & \\
\hline Tr. TV & 111.91 & 118.1 . & أ $1 \leqslant .1$. & 111.50 & $i v .00$ & I $\leqslant .7 \varepsilon$ & \multirow{2}{*}{ ربدون } \\
\hline$\cdot \varepsilon \cdot \pm$ & $\cdot . r q \pm$ & $\cdot \mu_{0} \pm$ & • & • & $\cdot r \leq \pm$ & $.11 \pm$ & \\
\hline \multicolumn{8}{|r|}{ * تأثير التغذية } \\
\hline IYI.Ar & 119.01 & $\mid 17.91$ & $\mid\{\leqslant\}$. & $1 \cdot .87$ & IV.07 & $\mid \leq . \wedge V$ & \multirow[t]{2}{*}{ مرة / يوميا } \\
\hline$\cdot \varepsilon \wedge \pm$ &.$\leqslant 0 \pm$ & $\cdot . \varepsilon 1 \pm$ & $. .49 \pm$ & $\cdot r^{T} \pm$ & $. .19 \pm$ & $\cdot \wedge_{ \pm}$ & \\
\hline IYI.AY & 119.90 & I IV.V. & $\mid 1 \leq .1 \leq$ & $11 \cdot .9$. & IV.rq & 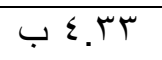 & \multirow{2}{*}{ مرتين| } \\
\hline$\cdot .7 r \pm$ & $.74 \pm$ & $\cdot . \leqslant \pm \pm$ &.$\leqslant 0 \pm$ & $\cdot . \leqslant T \pm$ & $\cdot r \cdot \pm$ & $. .1 V \pm$ & \\
\hline \multicolumn{8}{|c|}{ * تأثير الهرمونات } \\
\hline IYY.TE & $1 r \cdot .19$ & I IV. $\leqslant 9$ & I I $\leqslant$. YO $^{\circ}$ & 111.17 & $\mid \mathrm{l} \vee .0 \Lambda$ & $\mid \leq .71$ & \multirow{2}{*}{$\begin{array}{c}\text { PMSG + } \\
\text { GnRH }\end{array}$} \\
\hline$\cdot .7 r \pm$ & $\cdot .71 \pm$ & $\cdot \varepsilon \wedge \pm$ & $\cdot\{1 \pm$ & $\cdot r v \pm$ & $\cdot r \leq \pm$ & $. .1 \leq \pm$ & \\
\hline ITIr & 119.19 & $11 \mathrm{~V} .0$ & $11 \leq .9$ & $11 \cdot . \leqslant V$ & IV.YA & I \&.OV & \multirow{2}{*}{ PMSG } \\
\hline$\cdot\{1 \pm$ & $\cdot\{1 \pm$ & $\cdot .4 \pm$ & $\cdot . \varepsilon r \pm$ & $\cdot r \leq \pm$ & $. r T \pm$ & $. .10 \pm$ & \\
\hline \multicolumn{8}{|c|}{ ** تأثنير التداخل بين الرعي و التغذية و الهرمونات } \\
\hline 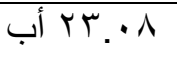 & 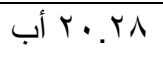 & (17.71 & 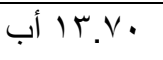 & $i l \cdot . \vee \wedge$ & IV. $\leqslant V$ & أ... & \multirow{2}{*}{1} \\
\hline $1 . \cdots \pm$ & $\cdot \wedge \wedge \pm$ & $1 . \cdot \leq \pm$ & $.94 \pm$ & $\cdot .71 \pm$ & $. r 4 \pm$ & $.1 r \pm$ & \\
\hline ع צ. آ أب & 19. 190 & (17.7. & 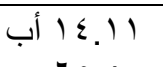 & أ $11 \cdot \varepsilon$ & $\mid \bar{I} V . \varepsilon V$ & 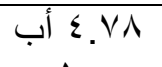 & \multirow{2}{*}{ r } \\
\hline$\because \wedge 1 \pm$ & $\cdot . \wedge 9 \pm$ & $.07 \pm$ & $. .70 \pm$ & $\cdot r 0 \pm$ & $\because r 9 \pm$ & $.10 \pm$ & \\
\hline I r $\left\{. V_{0}\right.$ & I KY.AO & $119 . \leqslant 0$ & 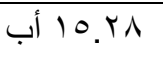 & أ.r. & 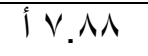 & 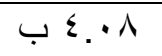 & \multirow{2}{*}{ r } \\
\hline $1.70 \pm$ & $1.19 \pm$ & $1 . r 1 \pm$ & $1 . r \pm \pm$ & $1 . v \pm$ & $\cdot V \leq \pm$ & 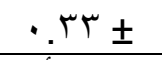 & \\
\hline 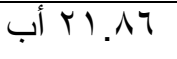 & . & 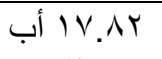 & أ. IV & I11.7V & 17.70 & r r. أب & \multirow{2}{*}{$\varepsilon$} \\
\hline $.0 r \pm$ & $\cdot \varepsilon 9 \pm$ & $\cdot \leqslant 7 \pm$ & $\cdot r \wedge \pm$ & $1 . .1 \pm$ & . & $\because r q \pm$ & \\
\hline ب. & & 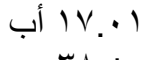 & $\mid 1 \leqslant .1\}$ & 111.11 & I v.o. & •. . ع أب & \multirow[t]{2}{*}{0} \\
\hline$\because V) \pm$ & $. .7 Y \pm$ & $\cdot r \wedge \pm$ & $\because \varepsilon 1 \pm$ & $\because \vee \vee \pm$ & $\cdot \therefore 9 \pm$ & $.11 \pm$ & \\
\hline • '. آ أب & 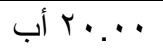 & 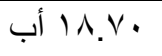 & I 117.r. & IIr.sr & IA.rV & I O.r. & \multirow{2}{*}{7} \\
\hline $1 . \varepsilon \cdot \pm$ & $r . \cdots \pm$ & $r . \cdots \pm$ & $1.1 \cdot \pm$ & $\cdot r r \pm$ & $\because \varepsilon V \pm$ & $\cdot r \cdot \pm$ & \\
\hline ب.90 & •r. & 0 ـ. Vا أب & r T. ا أب & I11.7V & IV.0 & ד. ₹. أب & \multirow[t]{2}{*}{ v } \\
\hline $1 . r \pm$ & $.94 \pm$ & $. .09 \pm$ & $\because V \cdot \pm$ & $\cdot . \wedge r \pm$ & $.7 r \pm$ & $\cdot \varepsilon r \pm$ & \\
\hline 19.90 & +r. & +. & . . & $|1 \cdot . \varepsilon|$ & IV.ry & 1 & \multirow[t]{2}{*}{$\Lambda$} \\
\hline$\because .01 \pm$ & $\cdot r \cdot \pm$ & $\cdot r \Lambda \pm$ & $\because \varepsilon \cdot \pm$ & $\cdot . Y \pm$ & • ${ }^{\top} \pm \pm$ & $\cdot \varepsilon r \pm$ & \\
\hline
\end{tabular}

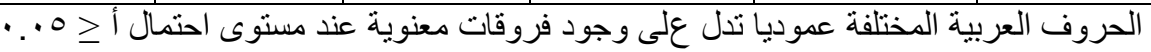

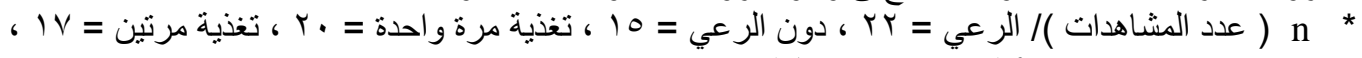
. 11 = PMSG ، 19 = PMSG+GnRH

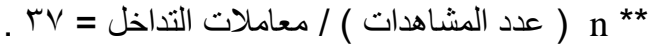

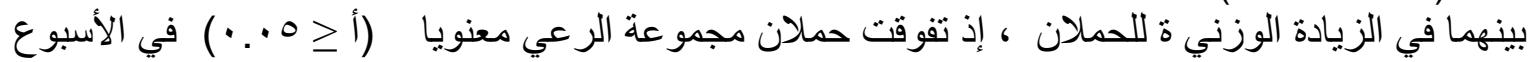

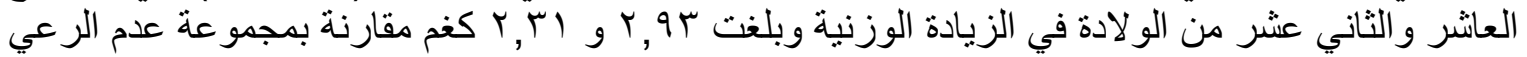

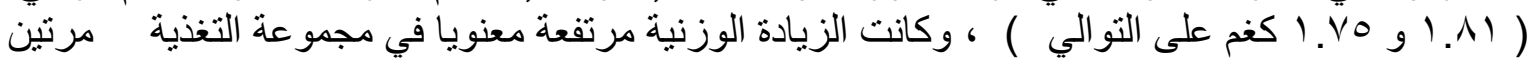

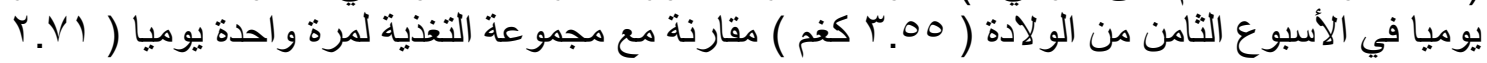




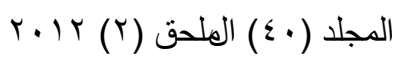
ISSN: 2224-9796 (Online)
ISSN: $1815-316 \mathrm{X}$ (print)
مجلة زر اعة الر افدين

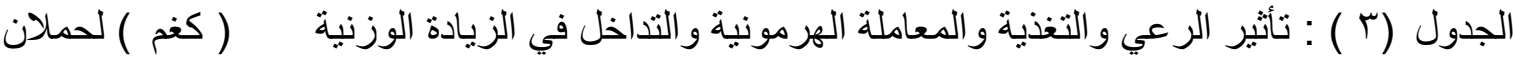

\begin{tabular}{|c|c|c|c|c|c|c|}
\hline الثاني عشرع & الأسبوع & الأسبوع & الأسبوع & الأسبوع الرابع & الأسبوع & المعاملاته الصفلت \\
\hline \multicolumn{7}{|r|}{ * تأثثير الرعي } \\
\hline$|T . M|$ & ir.qr & IT.IV & IT.TV & Ir.11 & IY.VE & \multirow[t]{2}{*}{ رعي } \\
\hline$. .1 V \pm$ & $\cdot r r_{ \pm}$ & $. Y T \pm$ & $\because r \wedge \pm$ & $.19 \pm$ & $\because M^{\top} \pm$ & \\
\hline 1.1. & (1.人1 & Ir... & IT.Ao & 15.79 & $|r . q|$ & ل بلون \\
\hline$\cdot .1 r \pm$ & $. .17 \pm$ & $\cdot Y \leq \pm$ & $\cdot r \cdot \pm$ & $.19 \pm$ & $\cdot r_{0} \pm$ & رعي \\
\hline \multicolumn{7}{|r|}{ * تاثير التنغذية } \\
\hline IT.Yr & I Y.7T & ו Y Y & $i r . \varepsilon r$ & IT.Y. & Ir.71 & \multirow[t]{2}{*}{ مرة / يوميل } \\
\hline $.11 \pm$ & $\cdot r^{r} \pm$ & $\cdot Y T \pm$ & $\cdot r \leqslant \pm$ & $\cdot Y I \pm$ & $\cdot r \cdot \pm$ & \\
\hline $\mid 1.91$ & I Y.ro & 14.00 & IT.TE & $|4.7|$ & I r.97 & \multirow{2}{*}{ مرتين| } \\
\hline $.1 \leq \pm$ & $\cdot . r \Lambda \pm$ & $\cdot Y^{Y} \pm$ & $\cdot Y 7 \pm$ & $.11 \pm$ & $\cdot . Y 7 \pm$ & \\
\hline \multicolumn{7}{|c|}{ * تأثير الهرمونات } \\
\hline 14.10 & I Y.V. & IT.rT & $i r . \wedge$ & Ir.01 & í.q. & \multirow{2}{*}{$\begin{array}{r}\text { PMSG + } \\
\text { GnRH }\end{array}$} \\
\hline $.19 \pm$ & $. \Gamma_{0} \pm$ & $\cdot r 4 \pm$ & $\cdot r \wedge \pm$ & $\cdot r \cdot \pm$ & $\cdot r V \pm$ & \\
\hline$i r . r$ & IT.YT & I Y.97 & $|r .7|$ & 14.19 & $|r . v|$ & \multirow[t]{2}{*}{ PMSG } \\
\hline$. .1 \leqslant \pm$ & . ro \pm & $\cdot Y T \pm$ & I & $\cdot . r \cdot \pm$ & $\cdot .1 V \pm$ & \\
\hline \multicolumn{7}{|c|}{ ** تأثثير التداخل بين الرعي و التخذية والهرمونات } \\
\hline I r. & أ. & $|r .9|$ & $\mid$ ir.q| & اسب. أب & $i r . \Sigma V$ & \multirow{2}{*}{1} \\
\hline$\cdot . \leqslant \vee \pm$ & $\cdot .7 V \pm$ & $.0 \cdot \pm$ & $\cdot .7 \cdot \pm$ & $. .9 \pm$ & $\cdot . \leqslant 7 \pm$ & \\
\hline 11 أب أب & 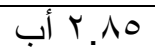 & I Y. $\varepsilon \wedge$ & $i \varepsilon . V$ & ب.07 & IY.TV & \multirow{2}{*}{ r } \\
\hline$\cdot r \cdot \pm$ & $. .0 r \pm$ & $\cdot . \Sigma \pm \pm$ & $\cdot .7 \leqslant \pm$ & $\cdot Y T \pm$ & $\cdot r) \pm$ & \\
\hline 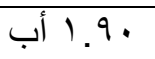 & Ir.s. & I $\leqslant .1 V$ & I r.qV & r r r r أب & I r.^. & \multirow{2}{*}{ 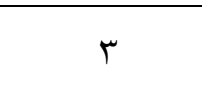 } \\
\hline 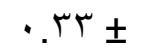 & $. .94 \pm$ & $\cdot . \leqslant \varepsilon \pm$ & r. $\mathrm{rV}_{ \pm}$ & $\cdot . \leqslant \leq \pm$ & $.07 \pm$ & \\
\hline ד Y. Y أب & أب أب أب & itr.70 & $i \uparrow . \wedge$. & 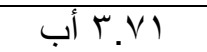 & I Y.r. & \multirow{2}{*}{$\varepsilon$} \\
\hline$\cdot . Y \tau \pm$ & $. M_{T} \pm$ & $.09 \pm$ & $\cdot . \varepsilon r \pm$ & $\cdot . \leqslant \pm$ & $\cdot . \leqslant \vee \pm$ & \\
\hline 99.1 أب & סV.1 أب & I r.Aq & $i r . .1$ & • . .بأب & ir.vq & \multirow{2}{*}{0} \\
\hline$\cdot r) \pm$ & $\cdot \mu^{\top} \pm$ & $.7 \varepsilon \pm$ & $.07 \pm$ & $\cdot \Sigma \vee \pm$ & $\cdot . \leqslant V \pm$ & \\
\hline . ا. ب. & •r. ب & I Y.o. & IT.VV & $1 \leqslant .10$ & IT.V & \multirow{2}{*}{7} \\
\hline$\cdot .7 \cdot \pm$ & \pm & $\cdot .9 \cdot \pm$ & $\cdot . \varepsilon r \pm$ & $.10 \pm$ & $\cdot V \vee \pm$ & \\
\hline ه. 1 أب & هV. أب & $|r . r|$ & I r.07 & أ & I r.VA & \multirow{2}{*}{ V } \\
\hline - YOE & $. r v \pm$ & $. Y T \pm$ & $.01 \pm$ & $. M^{\prime} \pm$ & $.79 \pm$ & \\
\hline VI I أب & •r. أب & Ir.IV & I r.sA & 10. & ir..9 & \multirow[b]{2}{*}{$\wedge$} \\
\hline$\cdot \mu^{r} \pm$ & $\cdot \mu^{\mu} \pm \pm$ & . TrE & $\cdot \Gamma \wedge \pm$ & $.1 \leqslant \pm$ & $\cdot .1 \wedge \pm$ & \\
\hline
\end{tabular}

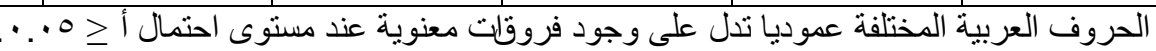

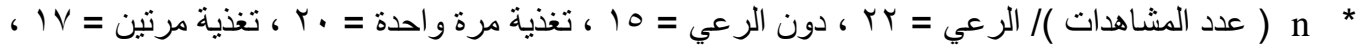
. $11=$ PMSG ، 19= PMSG+GnRH

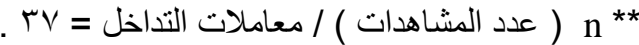

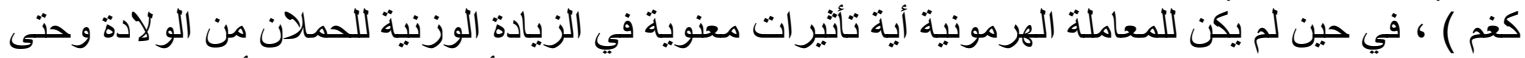

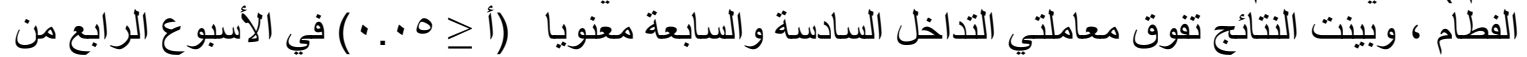

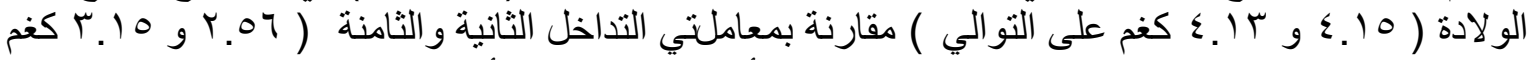

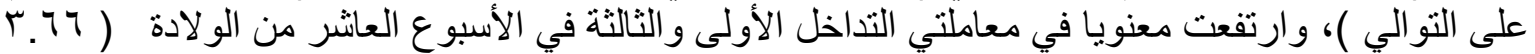

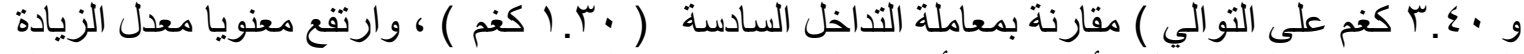

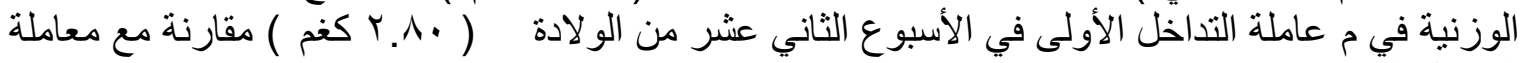

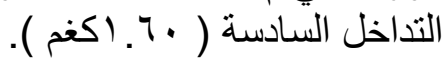


إن تفوق مو اليد مجموعة الرعي في وزن الجسم في الأسبوع العانشر حسابيا وفي الأسبوع الثاني لُاني

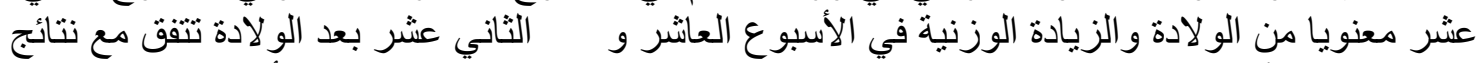
Braghieri

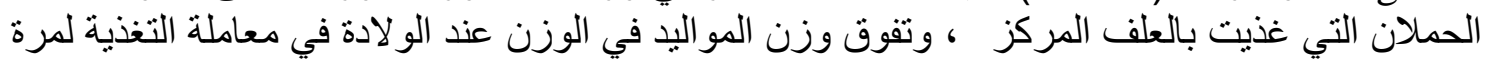

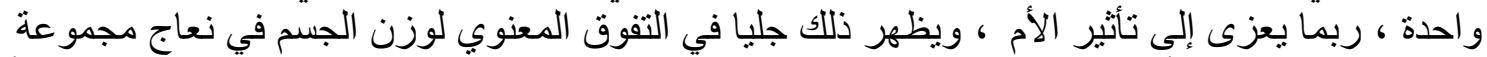

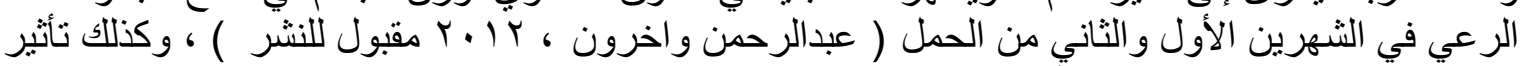

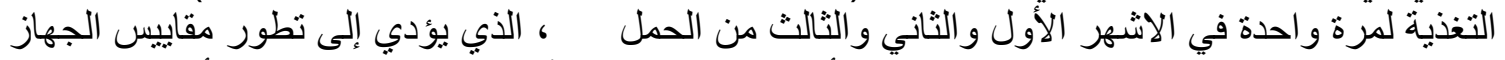

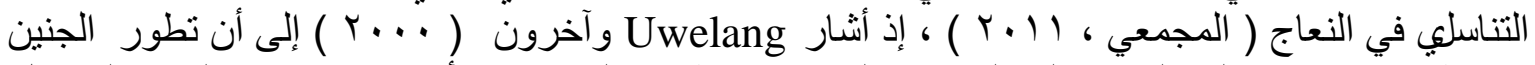

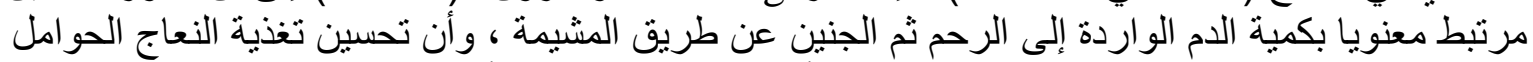

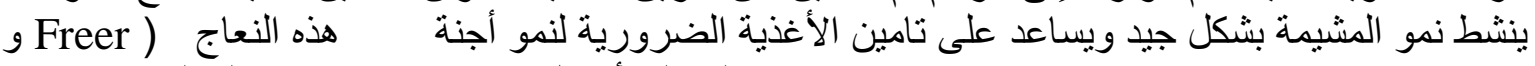

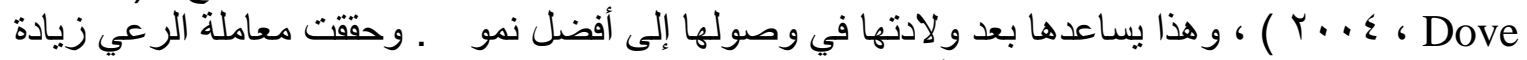

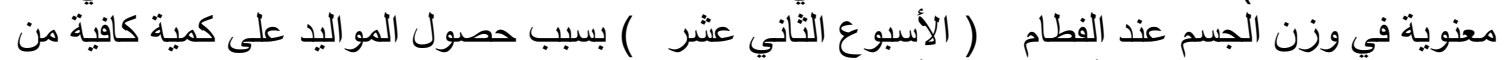

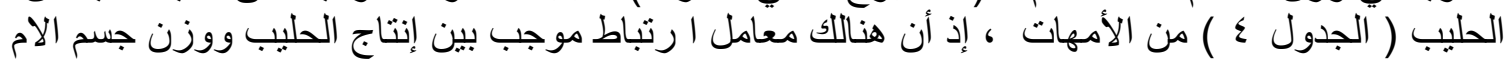

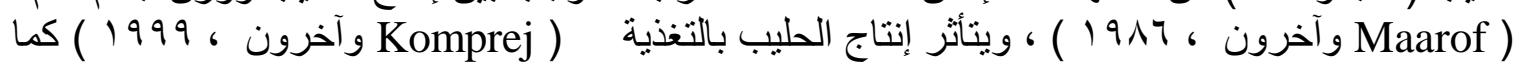

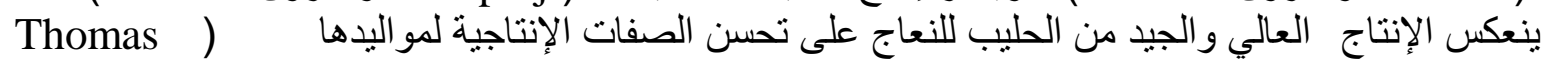

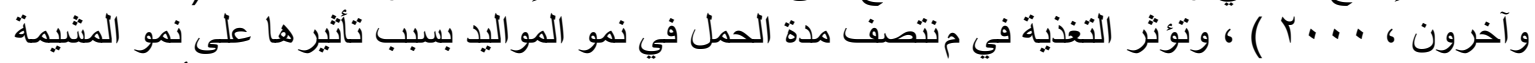

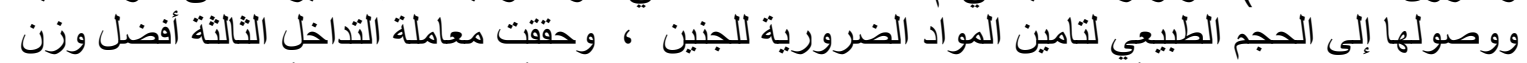

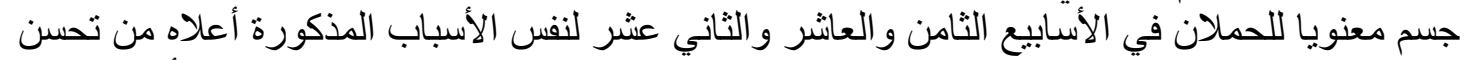

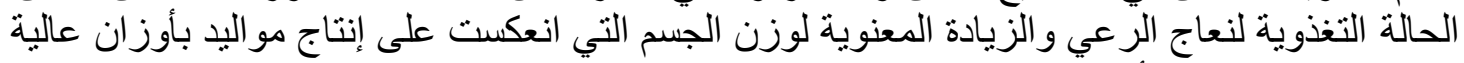

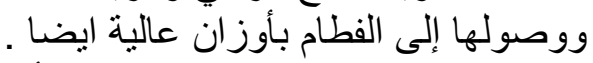

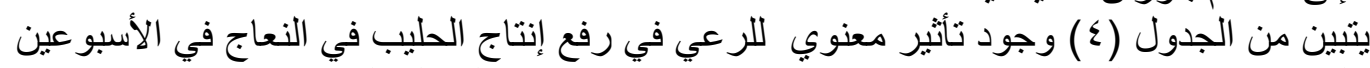

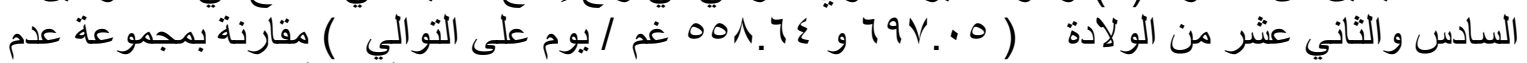

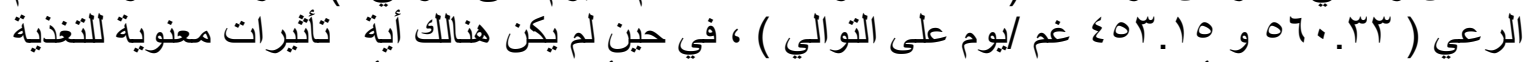

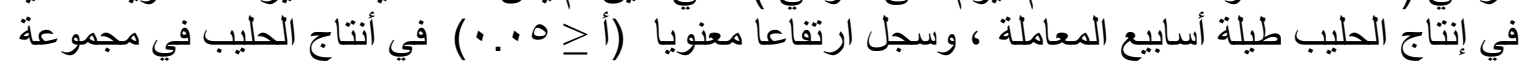

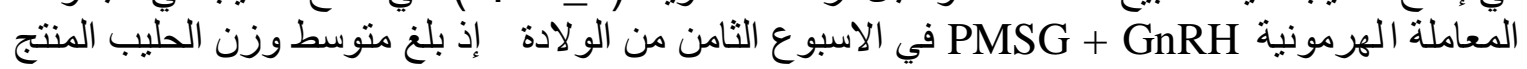

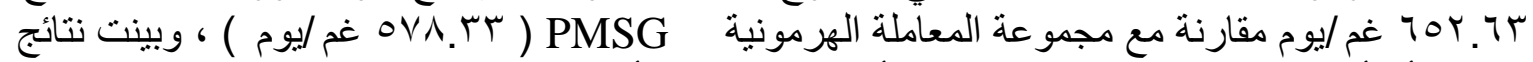

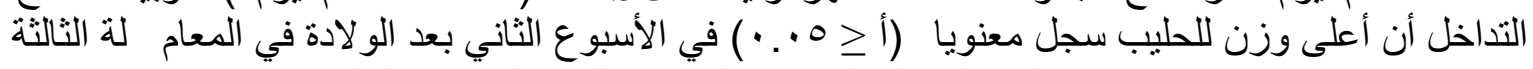

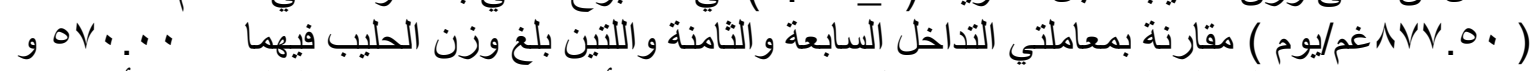
•.

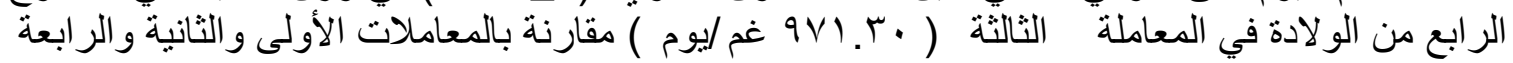

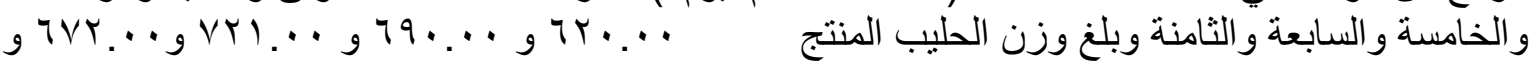

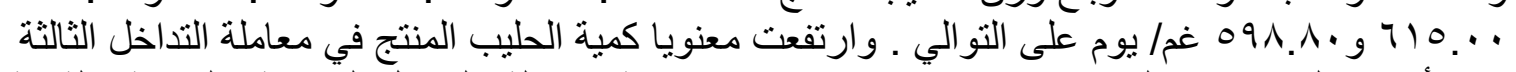

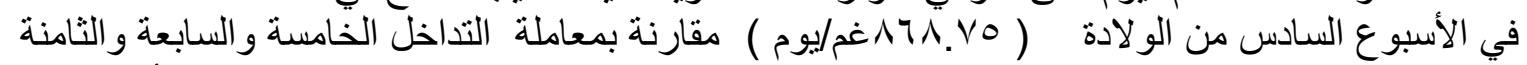

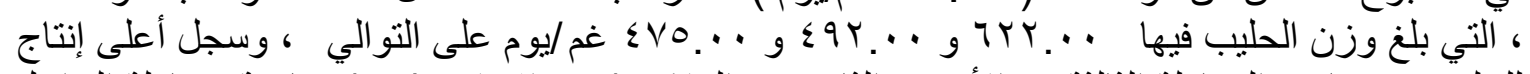

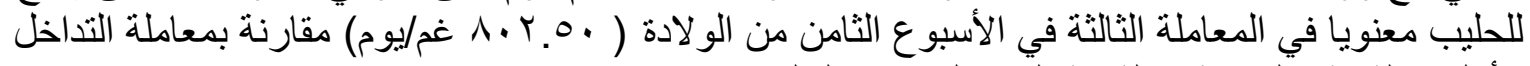

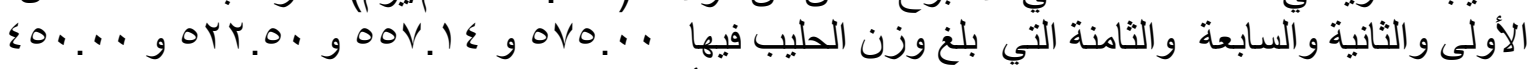

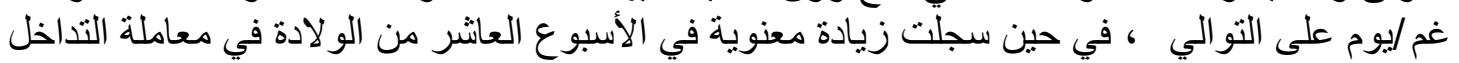

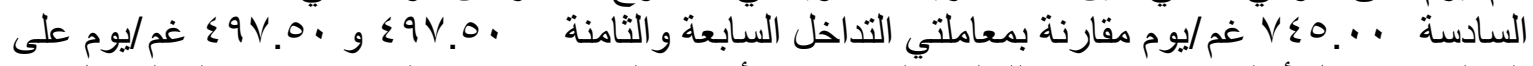

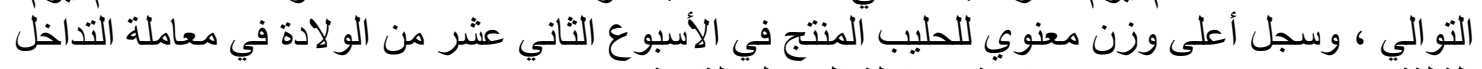

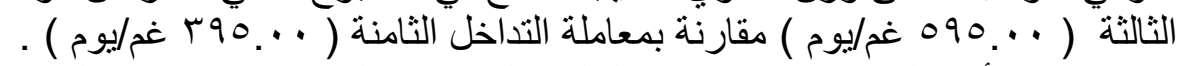

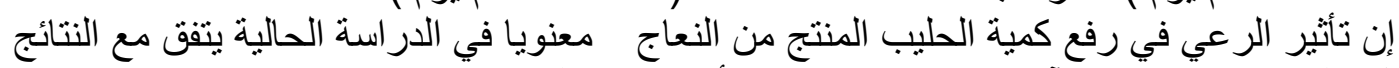

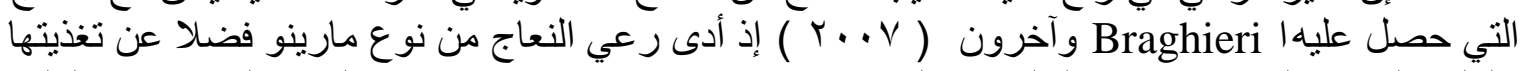

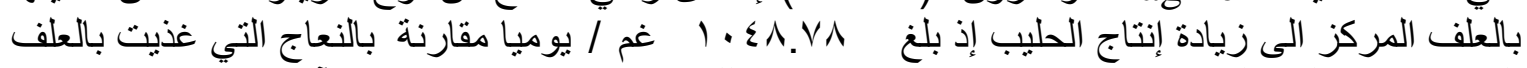

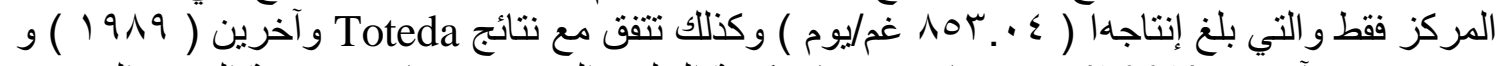
Ubertalle 


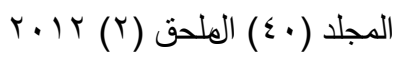
ISSN: 2224-9796 (Online)
ISSN: $1815-316 \mathrm{X}$ (print)
مجلة زر اعة الر افدين

الجدول(§) : تأثنر الرعي والتغذية والمعاملة الهرمونية والتداخل في إنتاج الحليب (غم /يوم ) للنعاج

\begin{tabular}{|c|c|c|c|c|c|c|}
\hline الثاني عشرع & الأسبوع & الأسبوع & الأسبوع & الأسبوع & الأسبوع & ماملات \\
\hline \multicolumn{7}{|r|}{ * تأثير الرعي } \\
\hline $1001.7 \varepsilon$ & $17 r 9.9$ & ITI.AT & 1798.0 & ivrq.9 & IVYo... & \multirow[t]{2}{*}{ رعي } \\
\hline$r \leq . Y \wedge \pm$ & $\Gamma \cdot . \wedge \pm$ & r. $17 \pm$ & I & 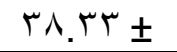 & ro.AV \pm & \\
\hline & $107 . .7 \mathrm{~V}$ & 109६... & س r. •070 ب & I I77.r & I ITV.rr & \multirow{2}{*}{ ربدي } \\
\hline$r \cdot .7 r \pm$ & & $\varepsilon r . r \cdot \pm$ & rV.q. \pm & $\leq Y .7 \leq \pm$ & or.rq & \\
\hline \multicolumn{7}{|r|}{ * تأثير التغذية } \\
\hline Iorg.ro & I Tr..o. & 1711.0. & 1701.20 & I \^乏.0. & $|v| \leq .0$. & \multirow[t]{2}{*}{ مرة / يو ميا } \\
\hline$Y V . \leq 1 \pm$ & T. I $1 \leq \pm$ & $1.70 \pm$ & ro.ru & ro. rᄉ \pm & $\varepsilon \cdot .1 \pm$ & \\
\hline I $\varepsilon \wedge \wedge . Y \leqslant$ & I OVA.Ar & $|7| \varepsilon .14$ & $|7 Y q . V|$ & IVTY.IA & I 77.... & \multirow{2}{*}{ مرتين| } \\
\hline & $r v .0 \pm$ & ד. ד. & $\varepsilon \Gamma . \varepsilon \cdot \pm$ & $\leq V . Y \leq \pm$ & $\leqslant \vee .9 \wedge \pm$ & \\
\hline \multicolumn{7}{|c|}{ * تأتثير الهرمونات } \\
\hline IOOKY & IOVT.17 & I & أ 707.0 & $I V \cdot 7.01$ & $|\vee| 1.01$ & \multirow{2}{*}{$\begin{array}{r}\text { PMSG + } \\
\text { GnRH }\end{array}$} \\
\hline ro. $V \pm$ & M. rA \pm & ro.r. \pm & $\varepsilon r .1 T \pm$ & $\varepsilon r . \leqslant \Lambda \pm$ & EY.YTI & \\
\hline $1019 . \leqslant \varepsilon$ & 174.11 & س r.rA. & I 7Y7.rq & IV...07 & $\mid 777.11$ & \multirow[t]{2}{*}{ PMSG } \\
\hline rᄉ. $r \varepsilon \pm$ & ro.A. \pm & rᄉ.ru & ro..9 & rq.0 \pm \pm & $\leqslant 0 . \leqslant \leq \pm$ & \\
\hline \multicolumn{7}{|c|}{ ** تأثثير التداخل بين الرعي والتغذية والهرمونات } \\
\hline 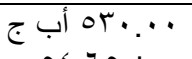 & r r.r. & & ا & P $^{\top r \cdot . \cdot}$ & 落 & \multirow{2}{*}{1} \\
\hline $0 \leqslant .70 \pm$ & $r 1 . r q \pm$ & $\leq 9.91 \pm$ & $71.9 \pm$ & $\leqslant 9.77 \pm$ & $0 \wedge . \vee \wedge \pm$ & \\
\hline 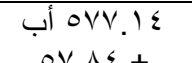 & ( & أ & 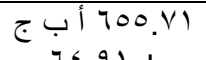 & 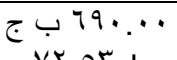 & r. & \multirow[t]{2}{*}{ r } \\
\hline $\begin{array}{l}\text { OV.A乏 } \pm \\
1090 . \cdots\end{array}$ & 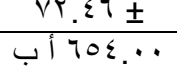 & $\begin{array}{l}\text { i li.r. } \\
\text { ro. }\end{array}$ & $\frac{1 \xi .91 \pm}{\mid \lambda \uparrow \lambda . V_{0}}$ & 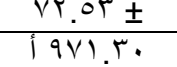 & 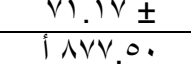 & \\
\hline 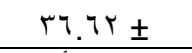 & $1 \cdots \leqslant 0 \pm$ & $r r . q 4 \pm$ & Tr. & $1 . . \Delta v \pm$ & $\leqslant 9.07 \pm$ & r \\
\hline 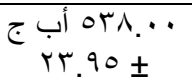 & 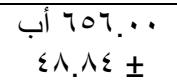 & 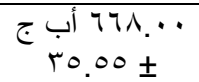 & 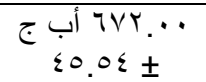 & 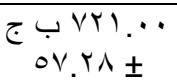 & 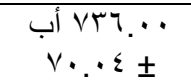 & $\varepsilon$ \\
\hline 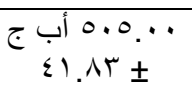 & 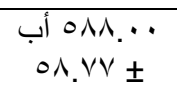 & $\begin{array}{l}\text { Tr...” } \\
\text { Vo.VI } \pm\end{array}$ & 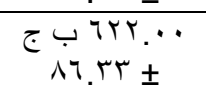 & 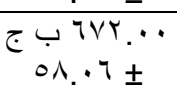 & $\begin{array}{l}\text { بqY.. } \\
99 . \vee 9 \pm \pm\end{array}$ & 0 \\
\hline 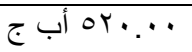 & $\mid v \leqslant 0 . .$. & 年 & .0. & ..... & 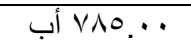 & \multirow[b]{2}{*}{7} \\
\hline$\varepsilon \cdot . \cdots \pm$ & $0 . \cdots \pm$ & $170 \ldots \pm$ & $11 \mathrm{r} .0 \cdot \pm$ & $1, \ldots \pm$ & $70 . \cdots \pm$ & \\
\hline 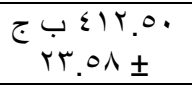 & $\begin{array}{c}\text { ب }\{9 \vee .0 . \\
\wedge \leqslant . \leqslant 9 \pm\end{array}$ & $\begin{array}{l}\text { Par.O. } \\
\text { IV.PI }\end{array}$ & 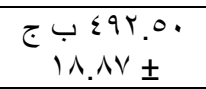 & $\begin{array}{l}710 . . \\
90.09 \pm\end{array}$ & 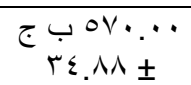 & V \\
\hline جr9o,.. & 列 & $د \leqslant 0 \cdot . . \cdot$ & ج $\leqslant$ V०... & $e^{09 \lambda . \wedge .}$ & ? $\leqslant r V .0$ & \multirow[t]{2}{*}{$\Lambda$} \\
\hline $15.01 \pm$ & $r 9.77 \pm$ & $r r . \wedge \cdot \pm$ & $r q .11 \pm$ & $\vee 9 . \Upsilon \wedge \pm$ & $0 \leqslant . \mu v \pm$ & \\
\hline
\end{tabular}

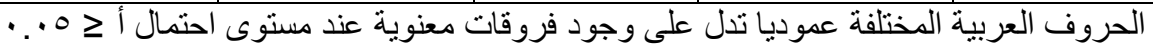

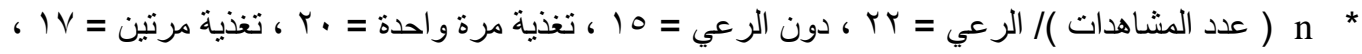
. $11=$ PMSG ، 19= PMSG+GnRH

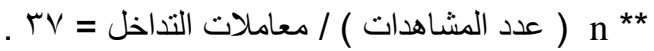

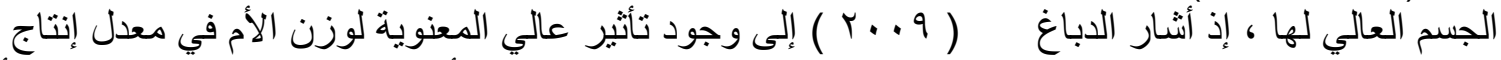

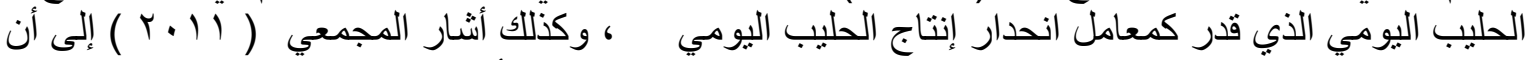

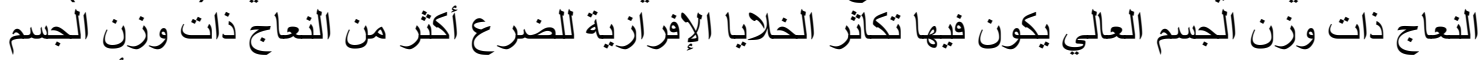

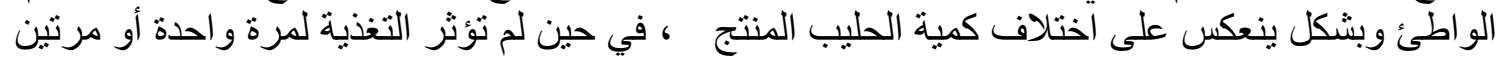

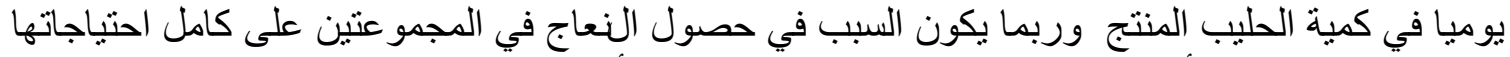

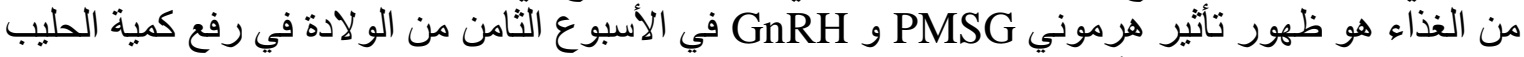

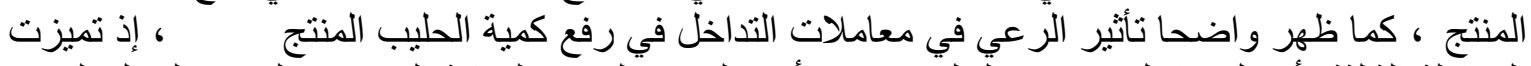

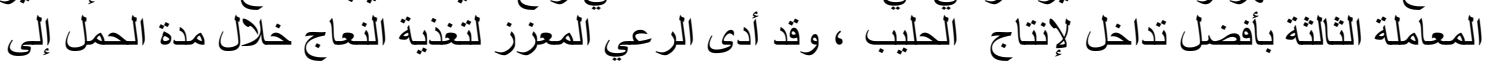

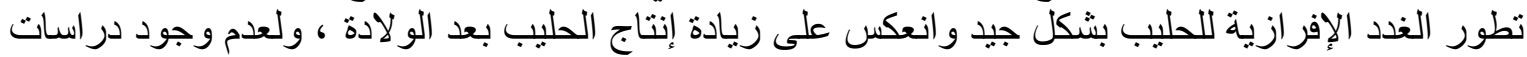

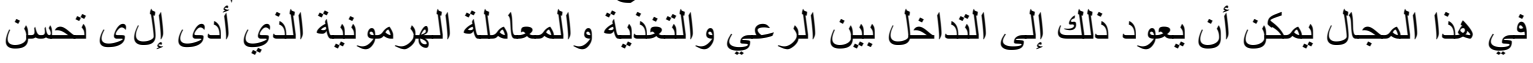




$$
\begin{aligned}
& \text { ～ISSN: 2224-9796 (Online) } \\
& \text { ISSN: } 1815-316 \mathrm{X} \text { (print) } \\
& \text { مجلة زر اعة الر افدين }
\end{aligned}
$$

\title{
EFFECT OF SOME FEEDING SYSTEMS AND HORMONAL TREATMENT ON REPRODUCTIVE PERFORMANCE AND LAMBS GROWTH IN AWASSI SHEEP
}

Sultan, KH. H. Abdul-Rahman, S.Y. Abdullah, R. KH.

Animal Resources Dept. , College of Agric. \& Forestry, Mosul Univ/Iraq

\begin{abstract}
The study was conducted on Eighty Awassi ewe lambs(10 month aged) to evaluate the effect of Grazing GR, Feeding frequency FF and hormonal treatment HT on ,reproductive performance and lambs growth, Estrous synchronization was conducted, Animal groups (10 ewes each ), 8 different treatments were applied including GR, FF, eCG and GnRH hormonal treatments. The results revealed that the Fertilization rate, Fertility\%, Lambing $\%$ at weaning and Barrenness $\%$ were better significantly $(\mathrm{P} \leq 0.01)$ in the $2^{\text {nd }}$ treatment $(\mathrm{GR}+\mathrm{ROD}+\mathrm{PMSG})$, while the results of the $3^{\text {rd }}$ treatment ( GR + RTD + PMSG \& GnRH ) and $5^{\text {th }}$ treatment ( without GR + ROD + PMSG \& GnRH ) in Litter size ( Higher value ) and Abortion ( Lower value ) were better significantly than other groups, the higher mortality \% of lambs was recorded in $6^{\text {th }}$ treatment ( without GR + ROD + PMSG ) group. The results also revealed a significant increase in lambs BW in GR treatment at $12^{\text {th }}$ week, and a significant increase in lambs birth weight in ROD treatment, while the best interaction effects in lambs weight were recorded in the $3^{\text {rd }}$ treatment ( GR + RTD + PMSG \& GnRH ) group at the $8^{\text {th }}, 10$ th and $12^{\text {th }}$ weeks and in the $6^{\text {th }}$ treatment ( without GR + ROD + PMSG ) at the $6^{\text {th }}$ week after lambing. The results also showed a significant increase in BG in ( GR ) group at the $10^{\text {th }}$ and $12^{\text {th }}$ weeks and in ( RTD ) group at the $8^{\text {th }}$ week, while the best interaction effects on BG was recorded in the $1^{\text {st }}$ treatment ( GR + ROD ++ PMSG \& GnRH ) group at $10^{\text {th }}$ and $12^{\text {th }}$ weeks after lambing. And in regard to milk production / day there is a significant increase in GR treatment at $6^{\text {th }}$ and $12^{\text {th }}$ weeks, and in (PMSG \& GnRH ) treatment at $8^{\text {th }}$ week, and the best interaction effects were recorded in the $3^{\text {rd }}$ treatment $(\mathrm{GR}+\mathrm{RTD}+\mathrm{PMSG} \& \mathrm{GnRH})$ group at $2^{\text {nd }}, 4^{\text {th }}, 6^{\text {th }}, 8^{\text {th }}, 12^{\text {th }}$ weeks after lambing. In conclusion grazing enhanced feeding state and improved the reproductive performance of ewes and milk production for lambs growth.

$$
\begin{aligned}
& \text { المصسادر }
\end{aligned}
$$

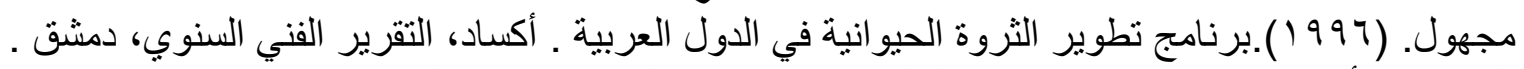

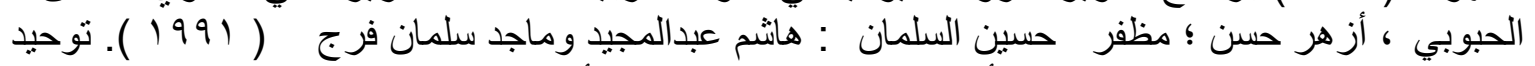

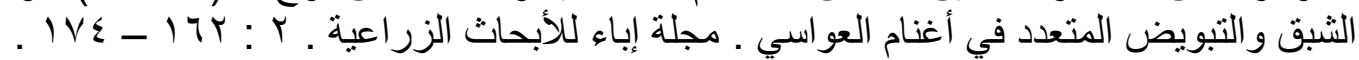

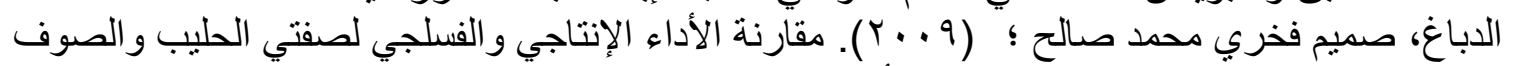

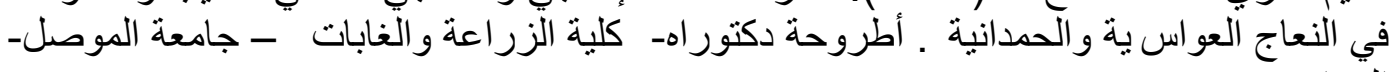

$$
\begin{aligned}
& \text { العر اق. }
\end{aligned}
$$

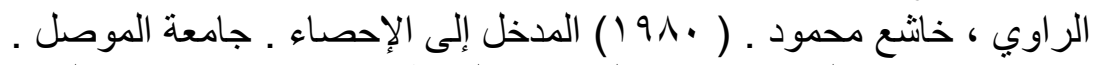

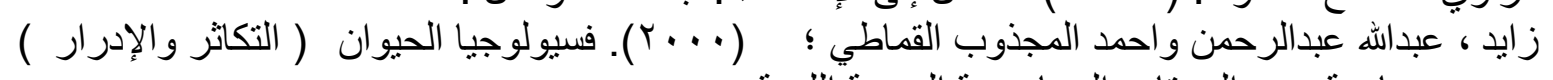

$$
\begin{aligned}
& \text { جامعة عمر المختار. الجماهيرية العربية الليبية. }
\end{aligned}
$$




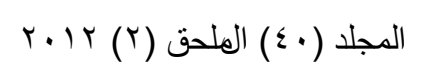

مجلة زر اعة الر افدين

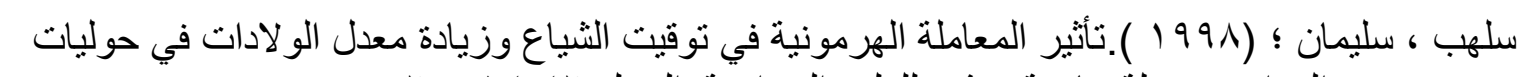

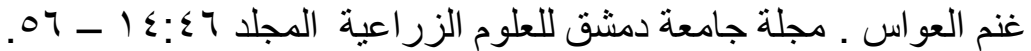

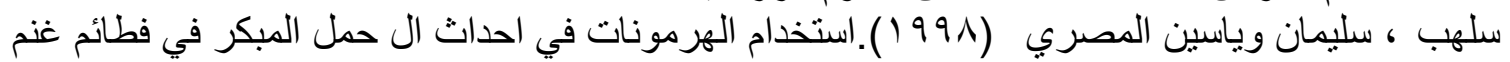

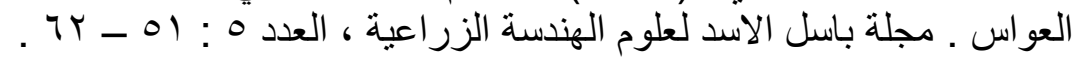

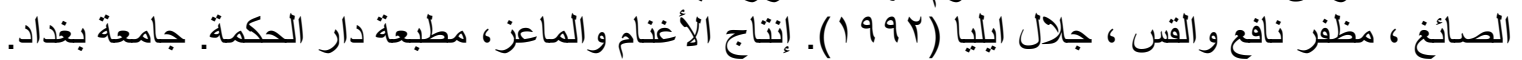

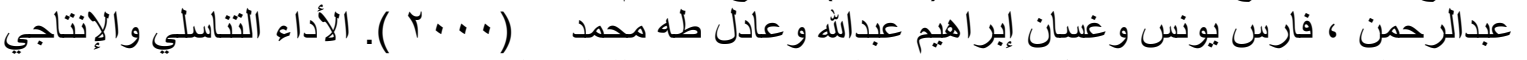

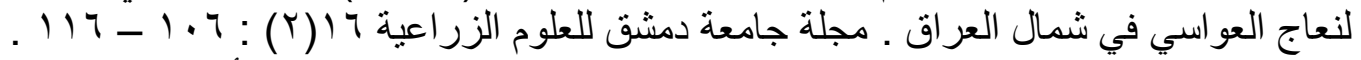

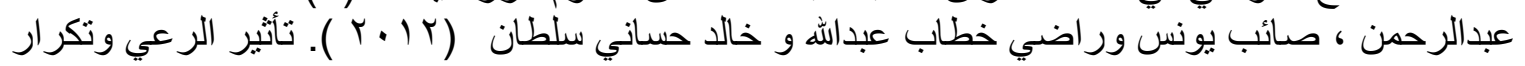

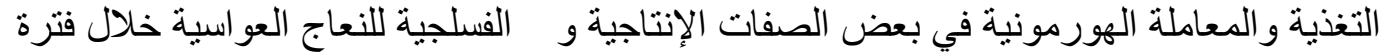

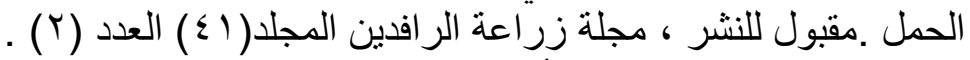

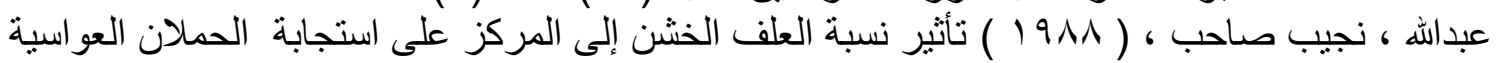

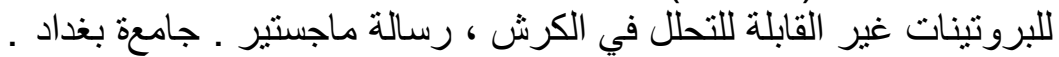

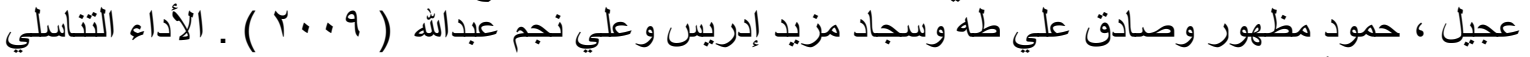

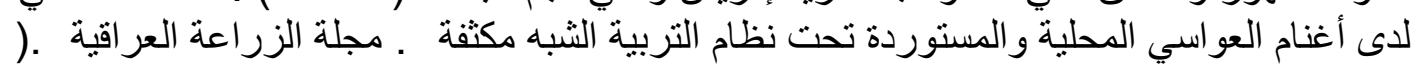

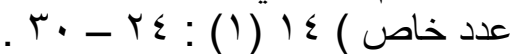

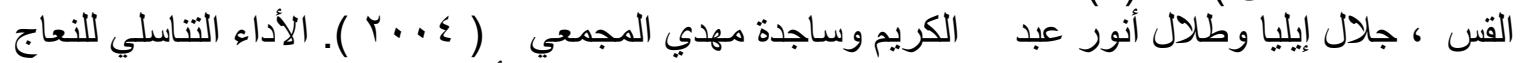

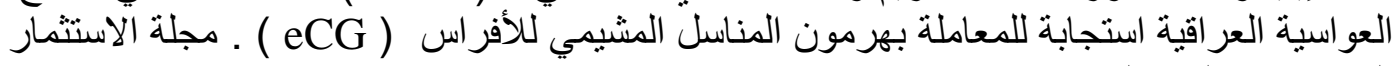

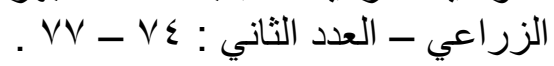

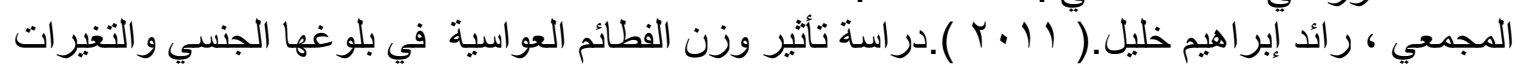

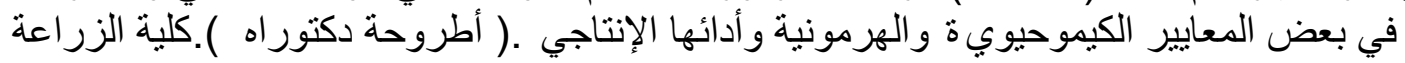

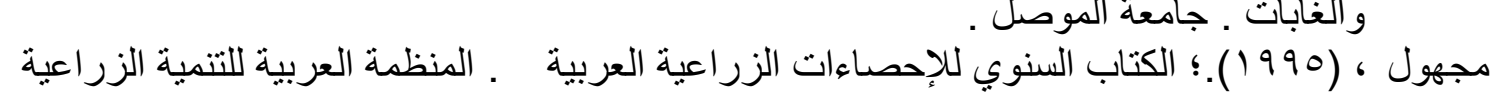

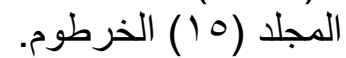

Bedawy, A.G.M. (2010). Effect Of Protein Level On The Attainment Of Puberty In Karadi Ram And Ewe Lambs And The Ovarian Response Of Ewes Treated With Lnsulin Dams Ph.D. Thesis, College of Agriculture, University of Duhok.

Braghieri, A, C. Pacelli, M. Verdone, A. Girolami, F. Napolitano.(2007). Effect of grazing and homeopathy on milk production and immunity of Merino derived ewes. Small Ruminant Research 69 (2007) 95-102.

Chao, L.M., K. Takayama, Y., Nakanishi, K., Hamana, M., Takagi, C., Kubota, and T., Kojima, (2008). Luteal lifespan and fertility after estrus synchronization in goats. J. Vet. Sci., 9, 95-101.

El-Hag, F. M., M. K. A. Ahmed, A. M. Salih, M. A. M Khair, B. Fadlalla, A. A. Ibnoaf and M. M. M. Ahmed, (2006). Supplementary feeding to improve desert sheep productivity under dry land farming. Trop. Sci., 47(1): 26-32.

Epstein H. (1985). Biology of reproduction, suckling regimes, growth, and development. In: The Awassi sheep with special reference to the improved dairy type. Rome: FAO. pp.81-140.

Freer, M.and H. Dove.(2004). Sheep Nutrition. Siro Publishing .Australia .

Hervé, V., F. Roy, J. Bertin, , F Guillou,. and M.C Maurel,., (2004). Anti-eCG antibodies generated in goats treated with eCG for the induction of ovulation modulate the LH and FSH bioactivities of eCG differently. Endocrinology, 145, 294-303.

Keskin, M., A.Gul, , S. Kaya, , M. Duru, , O. Gorgulu, S. Sahinler, and O.Bicer, (2007) .Effect of feed refreshing frequency on growth and carcass 


\section{～ISSN: 2224-9796 (Online) \\ ISSN: $1815-316 \mathrm{X}$ (print) \\ مجلة زر اعة الر افدين}

characteristics of Awassi lambs. South African Journal of Animal Science .37(4):318-323 .

Keskin, M., A.G.S Sahin,. and B, O. (2010). Effect of feed refreshing frequency on behavioral responses of Awassi lambs. Turkish Journal of Veterinary and Animal Science, 34 (4):333-33-8.

Komprej, A., M. Drobnic and D.Kompan (1999).Milk yield and milk traits Slovenian sheep breeds. Acta Agrar Kaposvariensis. 3 (2): 97 - 106 .

Kridli, R. T., A. Y. Abdulah. M. M. Shaker and Q. Al-Momani (2006b). Age at puberty and some biological parameters of Awassi and its first crosses with Charollais and Romanov rams. Ital. J. Sci. 5:193-202.

Lafi S. Q. A. Q. Talafha \& N. Giadinis \& E. Kalaitzakis \& K. ourliotis \& N. Panousis (2009) .Factors affecting the reproductive performance of Awassi sheep flocks in north-east of Jordan: An epidemiological study) . Trop Anim Health Prod (2009) 41:1755-1764.

Maarof, N. N., K.H. Juma, E. A. Araft and A. M. Chakmakchy.(1986). Evalution of factors affecting birth and weaning weight and milk production in Hamdani sheep. Wld. Rev. Anitn.Prod.22 : 51-55.

Pitono, A.D., E. Romijali, , M. Dally, , and R.M. Gatenby, , (1993). The effect of pregnant mare serum gonadotropin injection on ovulation rate in ewes. Jurnal- Penelitian-Peternaken-Sungei-Puith (JPPS), Indonesia, 1: 1-5.

Riesinberg, S., S.M Tillmann,., and B. Meinecke, , (2001). Ultrasonic study of follicular dynamics following superovulation in German Merino ewes. Theriogenology, 55, 847-865.

Anonymous.( 2002 ). Statistical Analysis Systems . Software, v.9, SAS Institute, Cary, NC.

Steel, R. G. D. and J. H. orrie, (1984). Principles and Procedures Of Statistics. 2nd Ed., McGraw-Hill Co., New York, USA.

Thomas, D. L., Y. M.Berger., and B. C. Mckusick.( 2000).East Friesian germplasm: Effect of milk production, lamb growth, and lamb survival. American Society of Animal Science.

Toteda, F., A.M Facciolongo,., R. Celi, , O. Montemurro, , (1989).Influenza del sistema di allevamento e dell'integrazione alimentare su alcune performance produttive e riproduttive della pecora. Arch. Vet. It. 40, 395-403.

Ubertalle, A., J. Errante, R. Fortina, , R. Ambrosoli, , (1991). Renneting ability and variations of some chemical, physical and biological parameters of sheep. In: Proceedings of the A.S.P.A. IX Congress, ISMEA, Roma, pp. 999-1008.

Ungerfeld, R., (2009). The induction of oestrus in ewes during the non-breeding season using pre-used CIDRs and oestradiol-17B treatment. Small Rumin. Res., 84, 129-131.

Uwelang, R., S. Baker., J. Khoury and K. K. Clark.(2000). Effects of chronic reduction in uterine blood flow on fetal and placental growth in the sheep. Am J. Physiol Regulatory Integrative Comp Physiol. 279: R53-R59. 